\title{
An integrated map of genetic variation from 1,092 human genomes
}

The 1000 Genomes Project Consortium*

By characterizing the geographic and functional spectrum of human genetic variation, the 1000 Genomes Project aims to build a resource to help to understand the genetic contribution to disease. Here we describe the genomes of 1,092 individuals from 14 populations, constructed using a combination of low-coverage whole-genome and exome sequencing. By developing methods to integrate information across several algorithms and diverse data sources, we provide a validated haplotype map of 38 million single nucleotide polymorphisms, 1.4 million short insertions and deletions, and more than 14,000 larger deletions. We show that individuals from different populations carry different profiles of rare and common variants, and that low-frequency variants show substantial geographic differentiation, which is further increased by the action of purifying selection. We show that evolutionary conservation and coding consequence are key determinants of the strength of purifying selection, that rare-variant load varies substantially across biological pathways, and that each individual contains hundreds of rare non-coding variants at conserved sites, such as motif-disrupting changes in transcription-factor-binding sites. This resource, which captures up to $98 \%$ of accessible single nucleotide polymorphisms at a frequency of $1 \%$ in related populations, enables analysis of common and low-frequency variants in individuals from diverse, including admixed, populations.

Recent efforts to map human genetic variation by sequencing exomes ${ }^{1}$ and whole genomes ${ }^{2-4}$ have characterized the vast majority of common single nucleotide polymorphisms (SNPs) and many structural variants across the genome. However, although more than $95 \%$ of common $(>5 \%$ frequency) variants were discovered in the pilot phase of the 1000 Genomes Project, lower-frequency variants, particularly those outside the coding exome, remain poorly characterized. Low-frequency variants are enriched for potentially functional mutations, for example, protein-changing variants, under weak purifying selection ${ }^{1,5,6}$. Furthermore, because low-frequency variants tend to be recent in origin, they exhibit increased levels of population differentiation ${ }^{6-8}$. Characterizing such variants, for both point mutations and structural changes, across a range of populations is thus likely to identify many variants of functional importance and is crucial for interpreting individual genome sequences, to help separate shared variants from those private to families, for example.

We now report on the genomes of 1,092 individuals sampled from 14 populations drawn from Europe, East Asia, sub-Saharan Africa and the Americas (Supplementary Figs 1 and 2), analysed through a combination of low-coverage $(2-6 \times)$ whole-genome sequence data, targeted deep (50-100 $\times$ ) exome sequence data and dense SNP genotype data (Table 1 and Supplementary Tables 1-3). This design was shown by the pilot phase ${ }^{2}$ to be powerful and cost-effective in discovering and genotyping all but the rarest SNP and short insertion and deletion (indel) variants. Here, the approach was augmented with statistical methods for selecting higher quality variant calls from candidates obtained using multiple algorithms, and to integrate SNP, indel and larger structural variants within a single framework (see

Table 1 | Summary of 1000 Genomes Project phase I data

\begin{tabular}{|c|c|c|c|}
\hline & Autosomes & Chromosome X & GENCODE regions* \\
\hline Samples & 1,092 & 1,092 & 1,092 \\
\hline Total raw bases (Gb) & 19,049 & 804 & 327 \\
\hline Mean mapped depth $(\times)$ & 5.1 & 3.9 & 80.3 \\
\hline \multicolumn{4}{|l|}{ SNPS } \\
\hline No. sites overall & $36.7 \mathrm{M}$ & $1.3 \mathrm{M}$ & $498 K$ \\
\hline Novelty rate $\dagger$ & $58 \%$ & $77 \%$ & $50 \%$ \\
\hline No. synonymous/non-synonymous/nonsense & NA & $4.7 / 6.5 / 0.097 \mathrm{~K}$ & $199 / 293 / 6.3 \mathrm{~K}$ \\
\hline Average no. SNPs per sample & $3.60 \mathrm{M}$ & $105 \mathrm{~K}$ & $24.0 \mathrm{~K}$ \\
\hline \multicolumn{4}{|l|}{ Indels } \\
\hline No. sites overall & $1.38 \mathrm{M}$ & $59 \mathrm{~K}$ & 1,867 \\
\hline Novelty rate $\dagger$ & $62 \%$ & $73 \%$ & $54 \%$ \\
\hline No. inframe/frameshift & NA & $19 / 14$ & $719 / 1,066$ \\
\hline Average no. indels per sample & $344 \mathrm{~K}$ & $13 \mathrm{~K}$ & 440 \\
\hline \multicolumn{4}{|l|}{ Genotyped large deletions } \\
\hline No. sites overall & $13.8 \mathrm{~K}$ & 432 & 847 \\
\hline Novelty rate $\uparrow$ & $54 \%$ & $54 \%$ & $50 \%$ \\
\hline Average no. variants per sample & 717 & 26 & 39 \\
\hline
\end{tabular}

NA, not applicable.

* Autosomal genes only.

$\uparrow$ Compared with dbSNP release 135 (Oct 2011), excluding contribution from phase I 1000 Genomes Project (or equivalent data for large deletions).

* Lists of participants and their affiliations appear at the end of the paper. 


\section{BOX I Constructing an integrated map of variation}

The 1,092 haplotype-resolved genomes released as phase I by the 1000 Genomes Project are the result of integrating diverse data from multiple technologies generated by several centres between 2008 and 2010. The Box 1 Figure describes the process leading from primary data production to integrated haplotypes.

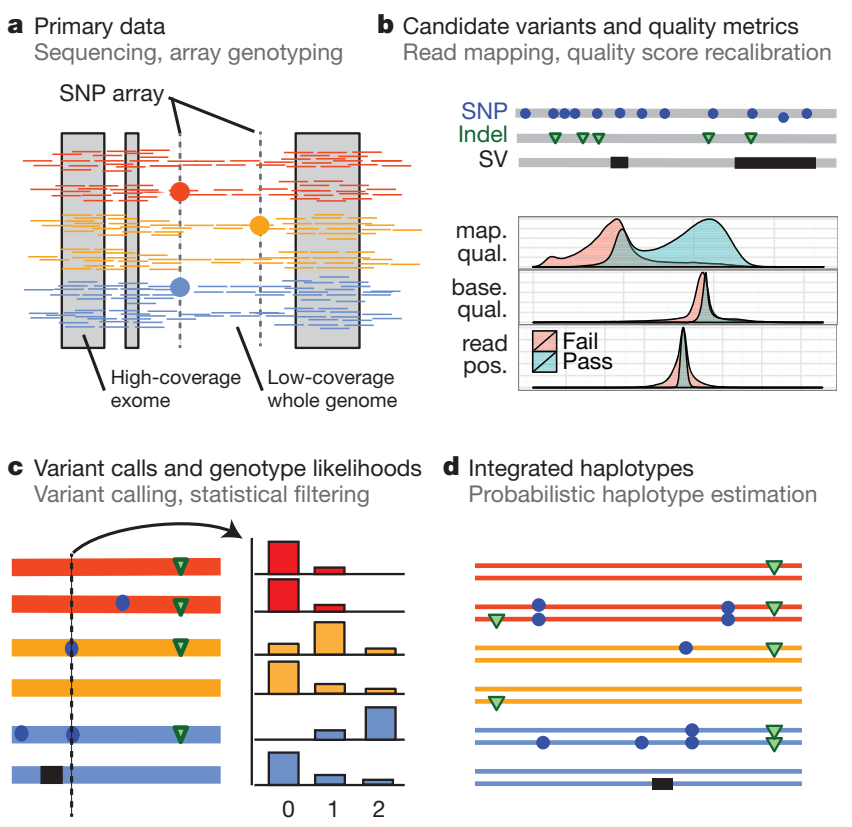

a, Unrelated individuals (see Supplementary Table 10 for exceptions) were sampled in groups of up to 100 from related populations (Wright's $F_{\mathrm{ST}}$ typically $<1 \%$ ) within broader geographical or ancestry-based groups ${ }^{2}$. Primary data generated for each sample consist of low-coverage (average $5 \times$ ) whole-genome and high-coverage (average $80 \times$ across a consensus target of $24 \mathrm{Mb}$ spanning more than 15,000 genes) exome sequence data, and high density SNP array information. b, Following read-alignment, multiple algorithms were used to identify candidate variants. For each variant, quality metrics were obtained, including information about the uniqueness of the surrounding sequence (for example, mapping quality (map. qual.)), the quality of evidence supporting the variant (for example, base quality (base. qual.) and the position of variant bases within reads (read pos.)), and the distribution of variant calls in the population (for example, inbreeding coefficient). Machine-learning approaches using this multidimensional information were trained on sets of high-quality known variants (for example, the high-density SNP array data), allowing variant sites to be ranked in confidence and subsequently thresholded to ensure low FDR. c, Genotype likelihoods were used to summarize the evidence for each genotype at biallelic sites ( 0,1 or 2 copies of the variant) in each sample at every site. d, As the evidence for a single genotype is typically weak in the low-coverage data, and can be highly variable in the exome data, statistical methods were used to leverage information from patterns of linkage disequilibrium, allowing haplotypes (and genotypes) to be inferred.

Box 1 and Supplementary Fig. 1). Because of the challenges of identifying large and complex structural variants and shorter indels in regions of low complexity, we focused on conservative but high-quality subsets: biallelic indels and large deletions.

Overall, we discovered and genotyped 38 million SNPs, 1.4 million bi-allelic indels and 14,000 large deletions (Table 1). Several technologies were used to validate a frequency-matched set of sites to assess and control the false discovery rate (FDR) for all variant types. Where results were clear, 3 out of 185 exome sites (1.6\%), 5 out of 281 low-coverage sites (1.8\%) and 72 out of 3,415 large deletions (2.1\%) could not be validated (Supplementary Information and Supplementary Tables 4-9). The initial indel call set was found to have a high FDR (27 out of 76), which led to the application of further filters, leaving an implied FDR of 5.4\% (Supplementary Table 6 and Supplementary Information). Moreover, for $2.1 \%$ of low-coverage SNP and $18 \%$ of indel sites, we found inconsistent or ambiguous results, indicating that substantial challenges remain in characterizing variation in low-complexity genomic regions. We previously described the 'accessible genome': the fraction of the reference genome in which short-read data can lead to reliable variant discovery. Through longer read lengths, the fraction accessible has increased from $85 \%$ in the pilot phase to $94 \%$ (available as a genome annotation; see Supplementary Information), and 1.7 million low-quality SNPs from the pilot phase have been eliminated.

By comparison to external SNP and high-depth sequencing data, we estimate the power to detect SNPs present at a frequency of $1 \%$ in the study samples is $99.3 \%$ across the genome and $99.8 \%$ in the consensus exome target (Fig. 1a). Moreover, the power to detect SNPs at $0.1 \%$ frequency in the study is more than $90 \%$ in the exome and nearly $70 \%$ across the genome. The accuracy of individual genotype calls at heterozygous sites is more than $99 \%$ for common SNPs and $95 \%$ for SNPs at a frequency of $0.5 \%$ (Fig. 1b). By integrating linkage disequilibrium information, genotypes from low-coverage data are as accurate as those from high-depth exome data for SNPs with frequencies $>1 \%$. For very rare SNPs ( $\leq 0.1 \%$, therefore present in one or two copies), there is no gain in genotype accuracy from incorporating linkage disequilibrium information and accuracy is lower. Variation among samples in genotype accuracy is primarily driven by sequencing depth (Supplementary Fig. 3) and technical issues such as sequencing platform and version (detectable by principal component analysis; Supplementary Fig. 4), rather than by population-level characteristics. The accuracy of inferred haplotypes at common SNPs was estimated by comparison to SNP data collected on mother-father-offspring trios for a subset of the samples. This indicates that a phasing (switch) error is made, on average, every 300-400 kilobases (kb) (Supplementary Fig. 5).

A key goal of the 1000 Genomes Project was to identify more than $95 \%$ of SNPs at $1 \%$ frequency in a broad set of populations. Our current resource includes $\sim 50 \%, 98 \%$ and $99.7 \%$ of the SNPs with frequencies of $\sim 0.1 \%, 1.0 \%$ and $5.0 \%$, respectively, in $\sim 2,500 \mathrm{UK}$ sampled genomes (the Wellcome Trust-funded UK10K project), thus a

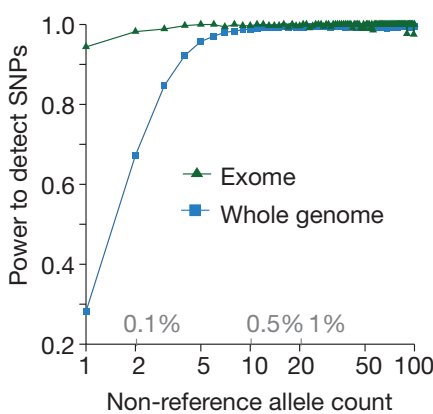

b

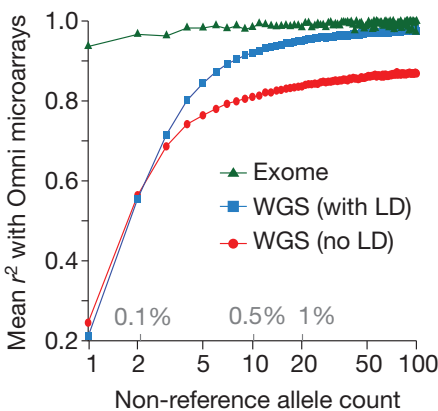

Figure $1 \mid$ Power and accuracy. a, Power to detect SNPs as a function of variant count (and proportion) across the entire set of samples, estimated by comparison to independent SNP array data in the exome (green) and whole genome (blue). b, Genotype accuracy compared with the same SNP array data as a function of variant frequency, summarized by the $r^{2}$ between true and inferred genotype (coded as 0,1 and 2) within the exome (green), whole genome after haplotype integration (blue), and whole genome without haplotype integration (red). LD, linkage disequilibrium; WGS, whole-genome sequencing. 
meeting this goal. However, coverage may be lower for populations not closely related to those studied. For example, our resource includes only $23.7 \%, 76.9 \%$ and $99.3 \%$ of the SNPs with frequencies of $\sim 0.1 \%$, $1.0 \%$ and $5.0 \%$, respectively, in $\sim 2,000$ genomes sequenced in a study of the isolated population of Sardinia (the SardiNIA study).

\section{Genetic variation within and between populations}

The integrated data set provides a detailed view of variation across several populations (illustrated in Fig. 2a). Most common variants ( $94 \%$ of variants with frequency $\geq 5 \%$ in Fig. 2 a) were known before the current phase of the project and had their haplotype structure mapped through earlier projects ${ }^{2,9}$. By contrast, only $62 \%$ of variants in the range $0.5-5 \%$ and $13 \%$ of variants with frequencies of $\leq 0.5 \%$ had been described previously. For analysis, populations are grouped by the predominant component of ancestry: Europe (CEU (see Fig. 2a for definitions of this and other populations), TSI, GBR, FIN and IBS), Africa (YRI, LWK and ASW), East Asia (CHB, JPT and CHS) and the Americas (MXL, CLM and PUR). Variants present at $10 \%$ and above across the entire sample are almost all found in all of the populations studied. By contrast, $17 \%$ of low-frequency variants in the range $0.5-5 \%$ were observed in a single ancestry group, and $53 \%$ of rare variants at $0.5 \%$ were observed in a single population (Fig. $2 \mathrm{~b}$ ). Within ancestry groups, common variants are weakly differentiated (most within-group estimates of Wright's fixation index $\left(F_{\mathrm{ST}}\right)$ are $<1 \%$; Supplementary Table 11), although below $0.5 \%$ frequency variants are up to twice as likely to be found within the same population compared with random samples from the ancestry group (Supplementary Fig. 6a). The degree of rare-variant differentiation varies between populations. For example, within Europe, the IBS and FIN populations carry excesses of rare variants (Supplementary Fig. $6 \mathrm{~b})$, which can arise through events such as recent bottlenecks ${ }^{10}$, 'clan' breeding structures ${ }^{11}$ and admixture with diverged populations ${ }^{12}$.

Some common variants show strong differentiation between populations within ancestry-based groups (Supplementary Table 12), many of which are likely to have been driven by local adaptation either directly or through hitchhiking. For example, the strongest differentiation between African populations is within an NRSF (neuron-restrictive silencer factor) transcription-factor peak (PANC1 cell line) ${ }^{13}$, upstream of ST8SIA 1 (difference in derived allele frequency LWK - YRI of 0.475 at rs7960970), whose product is involved in ganglioside generation ${ }^{14}$. Overall, we find a range of $17-343$ SNPs (fewest $=\mathrm{CEU}-\mathrm{GBR}$, most $=$ FIN - TSI) showing a difference in frequency of at least 0.25 between pairs of populations within an ancestry group.

The derived allele frequency distribution shows substantial divergence between populations below a frequency of $40 \%$ (Fig. 2c), such that individuals from populations with substantial African ancestry (YRI, LWK and ASW) carry up to three times as many low-frequency variants ( $0.5-5 \%$ frequency) as those of European or East Asian origin, reflecting ancestral bottlenecks in non-African populations ${ }^{15}$. However, individuals from all populations show an enrichment of rare variants $(<0.5 \%$ frequency), reflecting recent explosive increases in population size and the effects of geographic differentiation ${ }^{6,16}$. Compared with the expectations from a model of constant population size, individuals from all populations show a substantial excess of high-frequencyderived variants ( $>80 \%$ frequency).

Because rare variants are typically recent, their patterns of sharing can reveal aspects of population history. Variants present twice across the entire sample (referred to as $f_{2}$ variants), typically the most recent of informative mutations, are found within the same population in $53 \%$ of cases (Fig. 3a). However, between-population sharing identifies recent historical connections. For example, if one of the individuals carrying an $f_{2}$ variant is from the Spanish population (IBS) and the other is not (referred to as IBS-X), the other individual is more likely to come from the Americas populations (48\%, correcting for sample size) than from elsewhere in Europe (41\%). Within the East Asian populations, $\mathrm{CHS}$ and $\mathrm{CHB}$ show stronger $f_{2}$ sharing to each other a
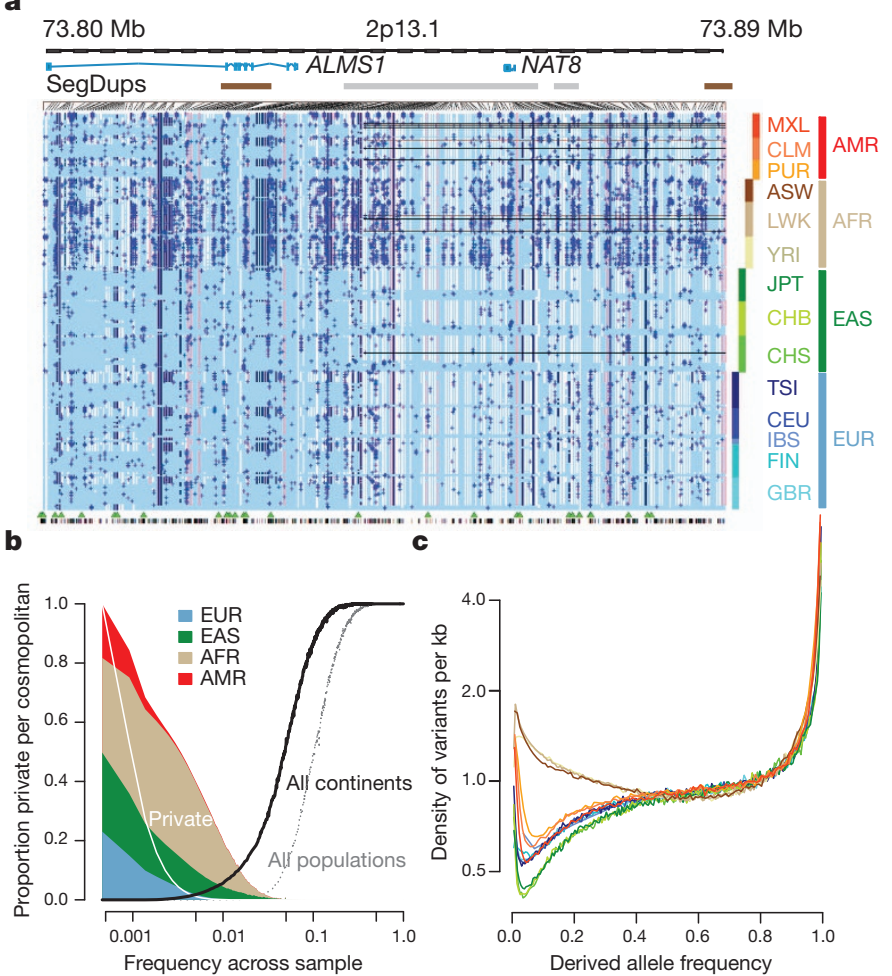

Figure $2 \mid$ The distribution of rare and common variants. a, Summary of inferred haplotypes across a 100-kb region of chromosome 2 spanning the genes ALMS1 and NAT8, variation in which has been associated with kidney disease ${ }^{45}$. Each row represents an estimated haplotype, with the population of origin indicated on the right. Reference alleles are indicated by the light blue background. Variants (non-reference alleles) above $0.5 \%$ frequency are indicated by pink (typed on the high-density SNP array), white (previously known) and dark blue (not previously known). Low frequency variants $(<0.5 \%)$ are indicated by blue crosses. Indels are indicated by green triangles and novel variants by dashes below. A large, low-frequency deletion (black line) spanning $N A T 8$ is present in some populations. Multiple structural haplotypes mediated by segmental duplications are present at this locus, including copy number gains, which were not genotyped for this study. Within each population, haplotypes are ordered by total variant count across the region. Population abbreviations: ASW, people with African ancestry in Southwest United States; CEU, Utah residents with ancestry from Northern and Western Europe; CHB, Han Chinese in Beijing, China; CHS, Han Chinese South, China; CLM, Colombians in Medellin, Colombia; FIN, Finnish in Finland; GBR, British from England and Scotland, UK; IBS, Iberian populations in Spain; LWK, Luhya in Webuye, Kenya; JPT, Japanese in Tokyo, Japan; MXL, people with Mexican ancestry in Los Angeles, California; PUR, Puerto Ricans in Puerto Rico; TSI, Toscani in Italia; YRI, Yoruba in Ibadan, Nigeria. Ancestry-based groups: AFR, African; AMR, Americas; EAS, East Asian; EUR, European. b, The fraction of variants identified across the project that are found in only one population (white line), are restricted to a single ancestry-based group (defined as in a, solid colour), are found in all groups (solid black line) and all populations (dotted black line). c, The density of the expected number of variants per kilobase carried by a genome drawn from each population, as a function of variant frequency (see Supplementary Information). Colours as in a. Under a model of constant population size, the expected density is constant across the frequency spectrum.

(58\% and $53 \%$ of $\mathrm{CHS}-\mathrm{X}$ and $\mathrm{CHB}-\mathrm{X}$ variants, respectively) than either does to JPT, but JPT is closer to CHB than to CHS (44\% versus $35 \%$ of JPT $-\mathrm{X}$ variants). Within African-ancestry populations, the ASW are closer to the YRI ( $42 \%$ of ASW $-\mathrm{X} f_{2}$ variants) than the LWK (28\%), in line with historical information ${ }^{17}$ and genetic evidence based on common $\mathrm{SNPs}^{18}$. Some sharing patterns are surprising; for example, $2.5 \%$ of the $f_{2}$ FIN-X variants are shared with YRI or LWK populations.

Independent evidence about variant age comes from the length of the shared haplotypes on which they are found. We find, as expected, 

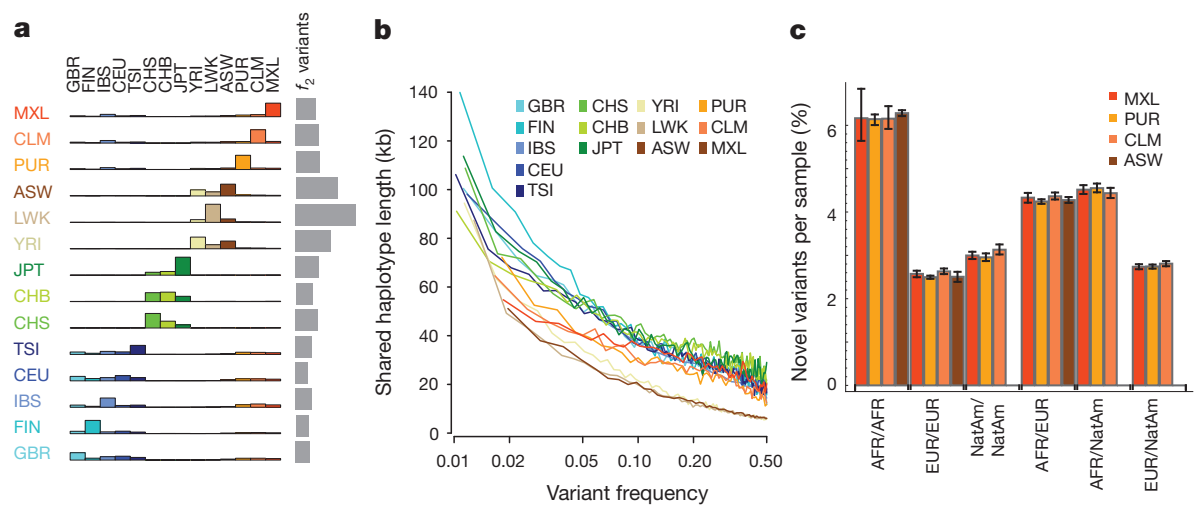

Figure $3 \mid$ Allele sharing within and between populations. a, Sharing of $f_{2}$ variants, those found exactly twice across the entire sample, within and between populations. Each row represents the distribution across populations for the origin of samples sharing an $f_{2}$ variant with the target population (indicated by the left-hand side). The grey bars represent the average number of $f_{2}$ variants carried by a randomly chosen genome in each population. $\mathbf{b}$, Median length of haplotype identity (excluding cryptically related samples and singleton variants, and allowing for up to two genotype errors) between two

a negative correlation between variant frequency and the median length of shared haplotypes, such that chromosomes carrying variants at $1 \%$ frequency share haplotypes of $100-150 \mathrm{~kb}$ (typically 0.08 $0.13 \mathrm{cM}$; Fig. $3 \mathrm{~b}$ and Supplementary Fig. 7a), although the distribution is highly skewed and $2-5 \%$ of haplotypes around the rarest SNPs extend over 1 megabase (Mb) (Supplementary Fig. 7b, c). Haplotype phasing and genotype calling errors will limit the ability to detect long shared haplotypes, and the observed lengths are a factor of 2-3 times shorter than predicted by models that allow for recent explosive growth $^{6}$ (Supplementary Fig. 7a). Nevertheless, the haplotype length for variants shared within and between populations is informative about relative allele age. Within populations and between populations in which there is recent shared ancestry (for example, through admixture and within continents), $f_{2}$ variants typically lie on long shared haplotypes (median within ancestry group $103 \mathrm{~kb}$; Supplementary Fig. 8). By contrast, between populations with no recent shared ancestry, $f_{2}$ variants are present on very short haplotypes, for example, an average of $11 \mathrm{~kb}$ for FIN - YRI $f_{2}$ variants (median between ancestry groups excluding admixture is $15 \mathrm{~kb}$ ), and are therefore likely to reflect recurrent mutations and chance ancient coalescent events.

To analyse populations with substantial historical admixture, statistical methods were applied to each individual to infer regions of the genome with different ancestries. Populations and individuals vary substantially in admixture proportions. For example, the MXL population contains the greatest proportion of Native American ancestry ( $47 \%$ on average compared with $24 \%$ in CLM and $13 \%$ in PUR), but the proportion varies from 3\% to 92\% between individuals (Supplementary Fig. 9a). Rates of variant discovery, the ratio of non-synonymous to synonymous variation and the proportion of variants that are new vary systematically between regions with different ancestries. Regions of Native American ancestry show less variation, but a higher fraction of the variants discovered are novel $3.0 \%$ of variants per sample; Fig. 3c) compared with regions of European ancestry (2.6\%). Regions of African ancestry show the highest rates of novelty (6.2\%) and heterozygosity (Supplementary Fig. 9b, c).

\section{The functional spectrum of human variation}

The phase I data enable us to compare, for different genomic features and variant types, the effects of purifying selection on evolutionary conservation $^{19}$, the allele frequency distribution and the level of differentiation between populations. At the most highly conserved coding sites, $85 \%$ of non-synonymous variants and more than $90 \%$ of stop-gain and splice-disrupting variants are below $0.5 \%$ in frequency, chromosomes that share variants of a given frequency in each population. Estimates are from 200 randomly sampled regions of $1 \mathrm{Mb}$ each and up to 15 pairs of individuals for each variant. c, The average proportion of variants that are new (compared with the pilot phase of the project) among those found in regions inferred to have different ancestries within ASW, PUR, CLM and MXL populations. Error bars represent $95 \%$ bootstrap confidence intervals. NatAm, Native American.

compared with $65 \%$ of synonymous variants (Fig. $4 a$ ). In general, the rare variant excess tracks the level of evolutionary conservation for variants of most functional consequence, but varies systematically between types (for example, for a given level of conservation enhancer variants have a higher rare variant excess than variants in transcription-factor motifs). However, stop-gain variants and, to a lesser extent, splice-site disrupting changes, show increased rare-variant excess whatever the conservation of the base in which they occur, as such mutations can be highly deleterious whatever the level of sequence conservation. Interestingly, the least conserved splice-disrupting variants show similar rare-variant loads to synonymous and noncoding regions, suggesting that these alternative transcripts are under very weak selective constraint. Sites at which variants are observed are typically less conserved than average (for example, sites with nonsynonymous variants are, on average, as conserved as third codon positions; Supplementary Fig. 10).

A simple way of estimating the segregating load arising from rare, deleterious mutations across a set of genes comes from comparing the
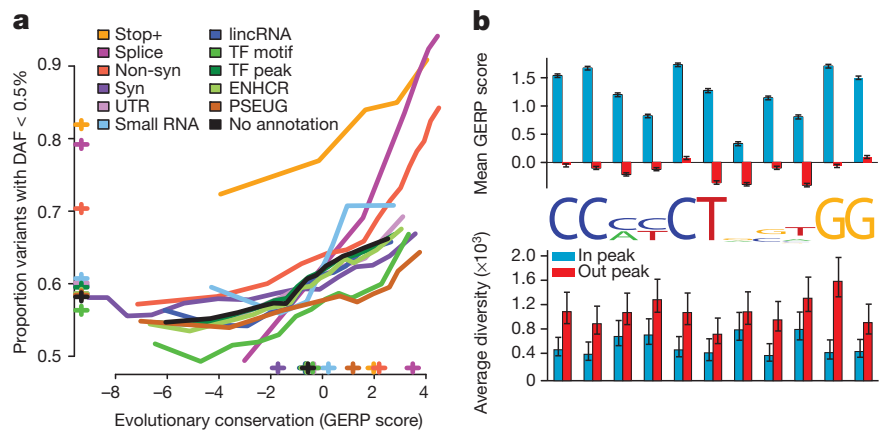

Figure $4 \mid$ Purifying selection within and between populations. a, The relationship between evolutionary conservation (measured by GERP score ${ }^{19}$ ) and rare variant proportion (fraction of all variants with derived allele frequency $(\mathrm{DAF})<0.5 \%)$ for variants occurring in different functional elements and with different coding consequences. Crosses indicate the average GERP score at variant sites ( $x$ axis) and the proportion of rare variants ( $y$ axis) in each category. ENHCR, enhancer; lincRNA, large intergenic non-coding RNA; non-syn, non-synonymous; PSEUG, pseudogene; syn, synonymous; TF, transcription factor. $\mathbf{b}$, Levels of evolutionary conservation (mean GERP score, top) and genetic diversity (per-nucleotide pairwise differences, bottom) for sequences matching the CTCF-binding motif within CTCF-binding peaks, as identified experimentally by ChIP-seq in the ENCODE project ${ }^{13}$ (blue) and in a matched set of motifs outside peaks (red). The logo plot shows the distribution of identified motifs within peaks. Error bars represent \pm 2 s.e.m. 
ratios of non-synonymous to synonymous variants in different frequency ranges. The non-synonymous to synonymous ratio among rare $(<0.5 \%)$ variants is typically in the range $1-2$, and among common variants in the range $0.5-1.5$, suggesting that $25-50 \%$ of rare non-synonymous variants are deleterious. However, the segregating rare load among gene groups in KEGG pathways ${ }^{20}$ varies substantially (Supplementary Fig. 11a and Supplementary Table 13). Certain groups (for example, those involving extracellular matrix (ECM)receptor interactions, DNA replication and the pentose phosphate pathway) show a substantial excess of rare coding mutations, which is only weakly correlated with the average degree of evolutionary conservation. Pathways and processes showing an excess of rare functional variants vary between continents (Supplementary Fig. 11b). Moreover, the excess of rare non-synonymous variants is typically higher in populations of European and East Asian ancestry (for example, the ECM-receptor interaction pathway load is strongest in European populations). Other groups of genes (such as those associated with allograft rejection) have a high non-synonymous to synonymous ratio in common variants, potentially indicating the effects of positive selection.

Genome-wide data provide important insights into the rates of functional polymorphism in the non-coding genome. For example, we consider motifs matching the consensus for the transcriptional repressor CTCF, which has a well-characterized and highly conserved binding motif ${ }^{21}$. Within CTCF-binding peaks experimentally defined by chromatin-immunoprecipitation sequencing (ChIP-seq), the average levels of conservation within the motif are comparable to third codon positions, whereas there is no conservation outside peaks (Fig. 4b). Within peaks, levels of genetic diversity are typically reduced $25-75 \%$, depending on the position in the motif (Fig. 4b). Unexpectedly, the reduction in diversity at some degenerate positions, for example, at position 8 in the motif, is as great as that at non-degenerate positions, suggesting that motif degeneracy may not have a simple relationship with functional importance. Variants within peaks show a weak but consistent excess of rare variation (proportion with frequency $<0.5 \%$ is $61 \%$ within peaks compared with $58 \%$ outside peaks; Supplementary Fig. 12), supporting the hypothesis that regulatory sequences contain substantial amounts of weakly deleterious variation.

Purifying selection can also affect population differentiation if its strength and efficacy vary among populations. Although the magnitude of the effect is weak, non-synonymous variants consistently show greater levels of population differentiation than synonymous variants for variants of frequencies of less than 10\% (Supplementary Fig. 13).

\section{Uses of 1000 Genomes Project data in medical genetics}

Data from the 1000 Genomes Project are widely used to screen variants discovered in exome data from individuals with genetic disorders ${ }^{22}$ and in cancer genome projects ${ }^{23}$. The enhanced catalogue presented here improves the power of such screening. Moreover, it provides a 'null expectation' for the number of rare, low-frequency and common variants with different functional consequences typically found in randomly sampled individuals from different populations.

Estimates of the overall numbers of variants with different sequence consequences are comparable to previous values ${ }^{1,20-22}$ (Supplementary Table 14). However, only a fraction of these are likely to be functionally relevant. A more accurate picture of the number of functional variants is given by the number of variants segregating at conserved positions (here defined as sites with a genomic evolutionary rate profiling $(\text { GERP })^{19}$ conservation score of $>2$ ), or where the function (for example, stop-gain variants) is strong and independent of conservation (Table 2). We find that individuals typically carry more than 2,500 nonsynonymous variants at conserved positions, $20-40$ variants identified as damaging ${ }^{24}$ at conserved sites and about 150 loss-of-function (LOF) variants (stop-gains, frameshift indels in coding sequence and disruptions to essential splice sites). However, most of these are common $(>5 \%)$ or low-frequency $(0.5-5 \%)$, such that the numbers of rare $(<0.5 \%)$ variants in these categories (which might be considered as pathological candidates) are much lower; 130-400 non-synonymous variants per individual, $10-20$ LOF variants, $2-5$ damaging mutations, and 1-2 variants identified previously from cancer genome sequencing ${ }^{25}$. By comparison with synonymous variants, we can estimate the excess of rare variants; those mutations that are sufficiently deleterious that they will never reach high frequency. We estimate that individuals carry an excess of 76-190 rare deleterious non-synonymous variants and up to $20 \mathrm{LOF}$ and disease-associated variants. Interestingly, the overall excess of low-frequency variants is similar to that of rare variants (Table 2). Because many variants contributing to disease risk are likely to be segregating at low frequency, we recommend that variant frequency be considered when using the resource to identify pathological candidates.

The combination of variation data with information about regulatory function ${ }^{13}$ can potentially improve the power to detect pathological

Table 2 | Per-individual variant load at conserved sites

\begin{tabular}{|c|c|c|c|c|c|}
\hline \multirow[t]{3}{*}{ Variant type } & \multicolumn{3}{|c|}{ Number of derived variant sites per individual } & \multirow[t]{3}{*}{ Excess rare deleterious } & \multirow[t]{3}{*}{ Excess low-frequency deleterious } \\
\hline & \multicolumn{3}{|c|}{ Derived allele frequency across sample } & & \\
\hline & $<0.5 \%$ & $0.5-5 \%$ & $>5 \%$ & & \\
\hline All sites & $30-150 \mathrm{~K}$ & $120-680 \mathrm{~K}$ & $3.6-3.9 \mathrm{M}$ & ND & ND \\
\hline Synonymous* & $29-120$ & $82-420$ & $1.3-1.4 \mathrm{~K}$ & ND & ND \\
\hline Non-synonymous* & 130-400 & 240-910 & $2.3-2.7 \mathrm{~K}$ & $76-190 \dagger$ & $77-130 \dagger$ \\
\hline Stop-gain* & $3.9-10$ & $5.3-19$ & 24-28 & $3.4-7.5+$ & $3.8-11 \dagger$ \\
\hline Stop-loss & $1.0-1.2$ & $1.0-1.9$ & $2.1-2.8$ & $0.81-1.1 \dagger$ & $0.80-1.0 \dagger$ \\
\hline HGMD-DM* & $2.5-5.1$ & $4.8-17$ & $11-18$ & $1.6-4.7 \dagger$ & $3.8-12 \dagger$ \\
\hline COSMIC* & $1.3-2.0$ & $1.8-5.1$ & $5.2-10$ & $0.93-1.6+$ & $1.3-2.0 \dagger$ \\
\hline Indel frameshift & $1.0-1.3$ & $11-24$ & $60-66$ & ND§ & $3.2-11 \dagger$ \\
\hline Indel non-frameshift & $2.1-2.3$ & $9.5-24$ & $67-71$ & ND§ & $0-0.73 \dagger$ \\
\hline Splice site donor & $1.7-3.6$ & $2.4-7.2$ & $2.6-5.2$ & $1.6-3.3 \dagger$ & $3.1-6.2 \dagger$ \\
\hline Splice site acceptor & $1.5-2.9$ & $1.5-4.0$ & $2.1-4.6$ & $1.4-2.6 \dagger$ & $1.2-3.3+$ \\
\hline UTR* & $120-430$ & $300-1,400$ & $3.5-4.0 \mathrm{~K}$ & $0-350$; & $0-1.2 \mathrm{~K}$ \\
\hline Non-coding RNA* & $3.9-17$ & 14-70 & $180-200$ & $0.62-2.6 t$ & $3.4-13 \div$ \\
\hline Motif gain in TF peak* & $4.7-14$ & $23-59$ & $170-180$ & $0-2.6+$ & $3.8-15 \dagger$ \\
\hline Motif loss in TF peak* & $18-69$ & $71-300$ & $580-650$ & $7.7-22 \%$ & $37-110 \ddagger$ \\
\hline Other conserved* & $2.0-9.9 \mathrm{~K}$ & $7.1-39 \mathrm{~K}$ & $120-130 \mathrm{~K}$ & ND & ND \\
\hline Total conserved & $2.3-11 \mathrm{~K}$ & $7.7-42 \mathrm{~K}$ & $130-150 \mathrm{~K}$ & $150-510$ & $250-1.3 \mathrm{~K}$ \\
\hline
\end{tabular}

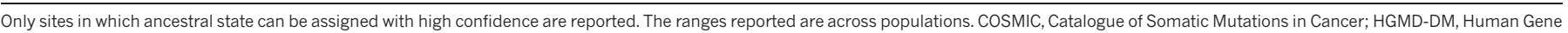
Mutation Database (HGMD) disease-causing mutations; TF, transcription factor; ND, not determined.

* Sites with GERP $>2$

$\dagger$ Using synonymous sites as a baseline.

† Using 'other conserved' as a baseline.

$\S$ Rare indels were filtered in phase I. 
non-coding variants. We find that individuals typically contain several thousand variants (and several hundred rare variants) in conserved (GERP conservation score >2) untranslated regions (UTR), noncoding RNAs and transcription-factor-binding motifs (Table 2). Within experimentally defined transcription-factor-binding sites, individuals carry 700-900 conserved motif losses (for the transcription factors analysed, see Supplementary Information), of which $18-69$ are rare $(<0.5 \%)$ and show strong evidence for being selected against. Motif gains are rarer ( $\sim 200$ per individual at conserved sites), but they also show evidence for an excess of rare variants compared with conserved sites with no functional annotation (Table 2). Many of these changes are likely to have weak, slightly deleterious effects on gene regulation and function.

A second major use of the 1000 Genomes Project data in medical genetics is imputing genotypes in existing genome-wide association studies (GWAS) ${ }^{26}$. For common variants, the accuracy of using the phase I data to impute genotypes at sites not on the original GWAS SNP array is typically $90-95 \%$ in non-African and approximately $90 \%$ in African-ancestry genomes (Fig. 5a and Supplementary Fig. 14a), which is comparable to the accuracy achieved with high-quality benchmark haplotypes (Supplementary Fig. 14b). Imputation accuracy is similar for intergenic SNPs, exome SNPs, indels and large deletions (Supplementary Fig. 14c), despite the different amounts of information about such variants and accuracy of genotypes. For lowfrequency variants (1-5\%), imputed genotypes have between $60 \%$ and $90 \%$ accuracy in all populations, including those with admixed ancestry (also comparable to the accuracy from trio-phased haplotypes; Supplementary Fig. 14b).

Imputation has two primary uses: fine-mapping existing association signals and detecting new associations. GWAS have had only a few examples of successful fine-mapping to single causal variants ${ }^{27,28}$, often because of extensive haplotype structure within regions of association $^{29,30}$. We find that, in Europeans, each previously reported GWAS signal ${ }^{31}$ is, on average, in linkage disequilibrium $\left(r^{2} \geq 0.5\right)$ with 56 variants: 51.5 SNPs and 4.5 indels. In $19 \%$ of cases at least one of these variants changes the coding sequence of a nearby gene (compared with $12 \%$ in control variants matched for frequency, distance to nearest gene and ascertainment in GWAS arrays) and in 65\% of cases
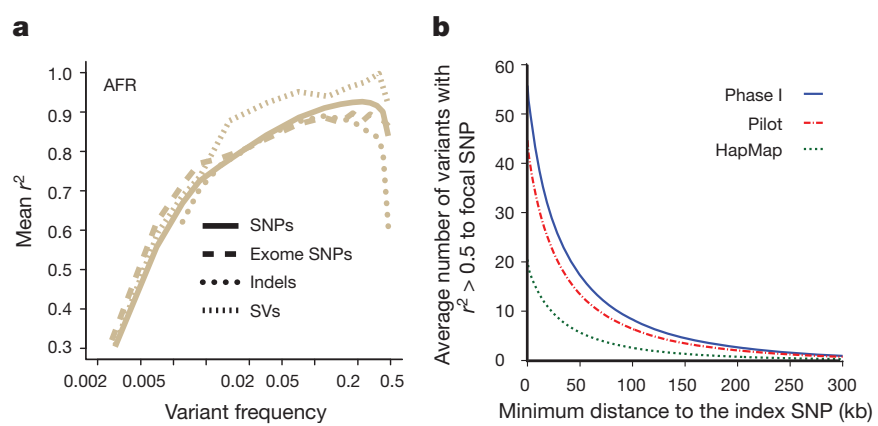

Figure 5 | Implications of phase I 1000 Genomes Project data for GWAS. a, Accuracy of imputation of genome-wide SNPs, exome SNPs and indels (using sites on the Illumina $1 \mathrm{M}$ array) into ten individuals of African ancestry (three LWK, four Masaai from Kinyawa, Kenya (MKK), two YRI), sequenced to high coverage by an independent technology ${ }^{3}$. Only indels in regions of high sequence complexity with frequency $>1 \%$ are analysed. Deletion imputation accuracy estimated by comparison to array data ${ }^{46}$ (note that this is for a different set of individuals, although with a similar ancestry, but included on the same plot for clarity). Accuracy measured by squared Pearson correlation coefficient between imputed and true dosage across all sites in a frequency range estimated from the $1000 \mathrm{Genomes}$ data. Lines represent whole-genome SNPs (solid), exome SNPs (long dashes), short indels (dotted) and large deletions (short dashes). SV, structural variants. $\mathbf{b}$, The average number of variants in linkage disequilibrium $\left(r^{2}>0.5\right.$ among EUR) to focal SNPs identified in GWAS $^{47}$ as a function of distance from the index SNP. Lines indicate the number of HapMap (green), pilot (red) and phase I (blue) variants. at least one of these is at a site with GERP $>2$ (68\% in matched controls). The size of the associated region is typically $<200 \mathrm{~kb}$ in length (Fig. 5b). Our observations suggest that trans-ethnic fine-mapping experiments are likely to be especially valuable: among the 56 variants that are in strong linkage disequilibrium with a typical GWAS signal, approximately 15 show strong disequilibrium across our four continental groupings (Supplementary Table 15). Our current resource increases the number of variants in linkage disequilibrium with each GWAS signal by $25 \%$ compared with the pilot phase of the project and by greater than twofold compared with the HapMap resource.

\section{Discussion}

The success of exome sequencing in Mendelian disease genetics ${ }^{32}$ and the discovery of rare and low-frequency disease-associated variants in genes associated with complex disease ${ }^{27,33,34}$ strongly support the hypothesis that, in addition to factors such as epistasis ${ }^{35,36}$ and geneenvironment interactions ${ }^{37}$, many other genetic risk factors of substantial effect size remain to be discovered through studies of rare variation. The data generated by the 1000 Genomes Project not only aid the interpretation of all genetic-association studies, but also provide lessons on how best to design and analyse sequencing-based studies of disease.

The use and cost-effectiveness of collecting several data types (lowcoverage whole-genome sequence, targeted exome data, SNP genotype data) for finding variants and reconstructing haplotypes are demonstrated here. Exome capture provides private and rare variants that are missed by low-coverage data (approximately $60 \%$ of the singleton variants in the sample were detected only from exome data compared with 5\% detected only from low-coverage data; Supplementary Fig. 15). However, whole-genome data enable characterization of functional non-coding variation and accurate haplotype estimation, which are essential for the analysis of cis-effects around genes, such as those arising from variation in upstream regulatory regions ${ }^{38}$. There are also benefits from integrating SNP array data, for example, to improve genotype estimation ${ }^{39}$ and to aid haplotype estimation where array data have been collected on additional family members. In principle, any sources of genotype information (for example, from array CGH) could be integrated using the statistical methods developed here.

Major methodological advances in phase I, including improved methods for detecting and genotyping variants ${ }^{40}$, statistical and machine-learning methods for evaluating the quality of candidate variant calls, modelling of genotype likelihoods and performing statistical haplotype integration ${ }^{41}$, have generated a high-quality resource. However, regions of low sequence complexity, satellite regions, large repeats and many large-scale structural variants, including copynumber polymorphisms, segmental duplications and inversions (which constitute most of the 'inaccessible genome'), continue to present a major challenge for short-read technologies. Some issues are likely to be improved by methodological developments such as better modelling of read-level errors, integrating de novo assembly ${ }^{42,43}$ and combining multiple sources of information to aid genotyping of structurally diverse regions ${ }^{40,44}$. Importantly, even subtle differences in data type, data processing or algorithms may lead to systematic differences in false-positive and false-negative error modes between samples. Such differences complicate efforts to compare genotypes between sequencing studies. Moreover, analyses that naively combine variant calls and genotypes across heterogeneous data sets are vulnerable to artefact. Analyses across multiple data sets must therefore either process them in standard ways or use meta-analysis approaches that combine association statistics (but not raw data) across studies.

Finally, the analysis of low-frequency variation demonstrates both the pervasive effects of purifying selection at functionally relevant sites in the genome and how this can interact with population history to lead to substantial local differentiation, even when standard metrics of structure such as $F_{\mathrm{ST}}$ are very small. The effect arises primarily 
because rare variants tend to be recent and thus geographically restricted $^{6-8}$. The implication is that the interpretation of rare variants in individuals with a particular disease should be within the context of the local (either geographic or ancestry-based) genetic background. Moreover, it argues for the value of continuing to sequence individuals from diverse populations to characterize the spectrum of human genetic variation and support disease studies across diverse groups. A further 1,500 individuals from 12 new populations, including at least 15 high-depth trios, will form the final phase of this project.

\section{METHODS SUMMARY}

All details concerning sample collection, data generation, processing and analysis can be found in the Supplementary Information. Supplementary Fig. 1 summarizes the process and indicates where relevant details can be found.

\section{Received 4 July; accepted 1 October 2012.}

1. Tennessen, J. A. et al. Evolution and functional impact of rare coding variation from deep sequencing of human exomes. Science 337, 64-69 (2012).

2. The 1000 Genomes Project Consortium. A map of human genome variation from population-scale sequencing. Nature 467, 1061-1073 (2010).

3. Drmanac, R. et al. Human genome sequencing using unchained base reads on self-assembling DNA nanoarrays. Science 327, 78-81 (2010).

4. Mills, R. E. et al. Mapping copy number variation by population-scale genome sequencing. Nature 470, 59-65 (2011).

5. Marth, G. T. et al. The functional spectrum of low-frequency coding variation. Genome Biol. 12, R84 (2011).

6. Nelson, M. R. et al. An abundance of rare functional variants in 202 drug target genes sequenced in 14,002 people. Science 337, 100-104 (2012).

7. Mathieson, I. \& McVean, G. Differential confounding of rare and common variants in spatially structured populations. Nature Genet. 44, 243-246 (2012).

8. Gravel, S. et al. Demographic history and rare allele sharing among human populations. Proc. Natl Acad. Sci. USA 108, 11983-11988 (2011).

9. The International HapMap Consortium. A second generation human haplotype map of over 3.1 million SNPs. Nature 449, 851-861 (2007).

10. Salmela, E. et al. Genome-wide analysis of single nucleotide polymorphisms uncovers population structure in Northern Europe. PLoS ONE 3, e3519 (2008).

11. Lupski, J. R., Belmont, J. W., Boerwinkle, E. \& Gibbs, R. A. Clan genomics and the complex architecture of human disease. Cell 147, 32-43 (2011).

12. Lawson, D. J., Hellenthal, G., Myers, S. \& Falush, D. Inference of population structure using dense haplotype data. PLoS Genet. 8, e1002453 (2012).

13. ENCODE Project Consortium. A user's guide to the encyclopedia of DNA elements (ENCODE). PLoS Biol. 9, e1001046 (2011).

14. Sasaki, K. et al. Expression cloning of a novel Galß(1-3/1-4)GIcNAc a2,3-sialyltransferase using lectin resistance selection. J. Biol. Chem. 268, 22782-22787 (1993)

15. Marth, G. et al. Sequence variations in the public human genome data reflect a bottlenecked population history. Proc. Natl Acad. Sci. USA 100, 376-381 (2003).

16. Keinan, A. \& Clark, A. G. Recent explosive human population growth has resulted in an excess of rare genetic variants. Science 336, 740-743 (2012).

17. Hall, G. M. Slavery and African Ethnicities in the Americas: Restoring the Links (Univ. North Carolina Press, 2005)

18. Bryc, K. etal. Genome-wide patterns of population structure and admixture in West Africans and African Americans. Proc. Natl Acad. Sci. USA 107, 786-791 (2010).

19. Davydov, E. V. et al. Identifying a high fraction of the human genome to be under selective constraint using GERP++. PLOS Comput. Biol. 6, e1001025 (2010).

20. Kanehisa, M., Goto, S., Sato, Y., Furumichi, M. \& Tanabe, M. KEGG for integration and interpretation of large-scale molecular data sets. Nucleic Acids Res. 40, D109-D114 (2012)

21. Kim, T. H. etal. Analysis of the vertebrate insulator protein CTCF-binding sites in the human genome. Cell 128, 1231-1245 (2007)

22. Bamshad, M. J. et al. Exome sequencing as a tool for Mendelian disease gene discovery. Nature Rev. Genet. 12, 745-755 (2011).

23. Cancer Genome Altas Research Network. Integrated genomic analyses of ovarian carcinoma. Nature 474, 609-615 (2011)

24. Stenson, P. D. et al. The Human Gene Mutation Database: 2008 update. Genome Med. 1, 13 (2009)

25. Forbes, S. A. et al. COSMIC: mining complete cancer genomes in the Catalogue of Somatic Mutations in Cancer. Nucleic Acids Res. 39, D945-D950 (2011).

26. Howie, B., Marchini, J. \& Stephens, M. Genotype imputation with thousands of genomes. G3 (Bethesda) 1, 457-470 (2011)

27. Sanna, S. et al. Fine mapping of five loci associated with low-density lipoprotein cholesterol detects variants that double the explained heritability. PLOS Genet. 7 , e1002198 (2011)

28. Gregory, A. P., Dendrou, C. A., Bell, J., McVean, G. \& Fugger, L. TNF receptor 1 genetic risk mirrors outcome of anti-TNF therapy in multiple sclerosis. Nature $\mathbf{4 8 8}$ 508-511 (2012).

29. Hassanein, M. T. etal. Fine mapping of the association with obesity at the FTO locus in African-derived populations. Hum. Mol. Genet. 19, 2907-2916 (2010).

30. Maller, J., The Wellcome Trust Case Control Consortium. Fine mapping of 14 loci identified through genome-wide association analyses. Nature Genet. (in the press).
31. Hindorff, L. A. et al. Potential etiologic and functional implications of genome-wide association loci for human diseases and traits. Proc. Natl Acad. Sci. USA 106, 9362-9367 (2009)

32. Bamshad, M. J. et al. The Centers for Mendelian Genomics: A new large-scale initiative to identify the genes underlying rare Mendelian conditions. Am. J. Med. Genet. A. (2012).

33. Momozawa, Y. et al. Resequencing of positional candidates identifies low frequency $I L 23 R$ coding variants protecting against inflammatory bowel disease. Nature Genet. 43, 43-47 (2011).

34. Raychaudhuri, S. et al. A rare penetrant mutation in $\mathrm{CFH}$ confers high risk of age-related macular degeneration. Nature Genet. 43, 1232-1236 (2011).

35. Strange, A. et al. A genome-wide association study identifies new psoriasis susceptibility loci and an interaction between HLA-C and ERAP1. Nature Genet. 42 , 985-990 (2010).

36. Zuk, O., Hechter, E., Sunyaev, S. R. \& Lander, E. S. The mystery of missing heritability: Genetic interactions create phantom heritability. Proc. Natl Acad. Sci. USA 109, 1193-1198 (2012).

37. Thomas, D. Gene-environment-wide association studies: emerging approaches. Nature Rev. Genet. 11, 259-272 (2010)

38. Degner, J. F. et al. DNase I sensitivity QTLs are a major determinant of human expression variation. Nature 482, 390-394 (2012).

39. Flannick, J. et al. Efficiency and power as a function of sequence coverage, SNP array density, and imputation. PLOS Comput. Biol. 8, e1002604 (2012).

40. Handsaker, R. E., Korn, J. M., Nemesh, J. \& McCarroll, S. A. Discovery and genotyping of genome structural polymorphism by sequencing on a population scale. Nature Genet. 43, 269-276 (2011).

41. Li, Y., Sidore, C., Kang, H. M., Boehnke, M. \& Abecasis, G. R. Low-coverage sequencing: implications for design of complex trait association studies. Genome Res. 21, 940-951 (2011)

42. Iqbal, Z., Caccamo, M., Turner, I., Flicek, P. \& McVean, G. De novo assembly and genotyping of variants using colored de Bruijn graphs. Nature Genet. 44, 226-232 (2012).

43. Simpson, J.T.\& Durbin, R. Efficient construction of an assembly string graph using the FM-index. Bioinformatics 26, i367-i373 (2010).

44. Sudmant, P. H. et al. Diversity of human copy number variation and multicopy genes. Science 330, 641-646 (2010).

45. Chambers, J. C. et al. Genetic loci influencing kidney function and chronic kidney disease. Nature Genet. 42, 373-375 (2010).

46. Conrad, D. F. et al. Origins and functional impact of copy number variation in the human genome. Nature 464, 704-712 (2010).

47. Hindorff, L. A. et al. A Catalog of Published Genome-Wide Association Studies. Available at http://www.genome.gov/gwastudies (accessed, September 2012).

Supplementary Information is available in the online version of the paper.

Acknowledgements We thank many people who contributed to this project: A. Naranjo, M. V. Parra and C. Duque for help with the collection of the Colombian samples; N. Kälin and F. Laplace for discussions; A. Schlattl and T. Zichner for assistance in managing data sets; E. Appelbaum, H. Arbery, E. Birney, S. Bumpstead, J. Camarata, J. Carey, G. Cochrane, M. DaSilva, S. Dökel, E. Drury, C. Duque, K. Gyaltsen, P. Jokinen, B. Lenz, S. Lewis, D. Lu, A. Naranjo, S. Ott, I. Padioleau, M. V. Parra, N. Patterson, A. Price, L. Sadzewicz, S. Schrinner, N. Sengamalay, J. Sullivan, F. Ta, Y. Vaydylevich, O. Venn, K. Watkins and A. Yurovsky for assistance, discussion and advice. We thank the people who generously contributed their samples, from these populations: Yoruba in Ibadan, Nigeria; the Han Chinese in Beijing, China; the Japanese in Tokyo, Japan; the Utah CEPH community; the Luhya in Webuye, Kenya; people with African ancestry in the Southwest United States; the Toscani in Italia; people with Mexican ancestry in Los Angeles, California; the Southern Han Chinese in China; the British in England and Scotland; the Finnish in Finland; the Iberian Populations in Spain; the Colombians in Medellin, Colombia; and the Puerto Ricans in Puerto Rico. This research was supported in part by Wellcome Trust grants WT098051 to R.M.D., M.E.H. and C.T.S.; WT090532/Z/09/Z, WT085475/Z/08/Z and WT095552/ Z/11/Z to P.Do.; WT086084/Z/08/Z and WT090532/Z/09/Z to G.A.M. WT089250/Z/ 09/Z to I.M.; WT085532AIA to P.F.; Medical Research Council grant G0900747(91070) to G.A.M.; British Heart Foundation grant RG/09/12/28096 to C.A.A. the National Basic Research Program of China ( 973 program no. 2011CB809201, 2011CB809202 and 2011CB809203); the Chinese 863 program (2012AA02A201); the National Natural Science Foundation of China (30890032. 31161130357); the Shenzhen Key Laboratory of Transomics Biotechnologies (CXB201108250096A); the Shenzhen Municipal Government of China (grants ZYC200903240080A and ZYC201105170397A); Guangdong Innovative Research Team Program (no. 2009010016); BMBF grant 01GS08201 to H.Le.; BMBF grant 0315428A to R.H.; the Max Planck Society; Swiss National Science Foundation 31003A_130342 to E.T.D.; Swiss National Science Foundation NCCR 'Frontiers in Genetics' grant to E.T.D.; Louis Jeantet Foundation grant to E.T.D.; Biotechnology and Biological Sciences Research Council (BBSRC) grantBB/I021213/1 to A.R.L.'German Research Foundation (Emmy Noether Fellowship KO 4037/1-1) to J.O.K.; Netherlands Organization for Scientific Research VENI grant 639.021 .125 to K.Y.; Beatriu de Pinos Program grants 2006BP-A 10144 and 2009BP-B 00274 to M.V.; Israeli Science Foundation grant 04514831 to E.H.; Genome Québec and the Ministry of Economic Development, Innovation and Trade grant PSR-SIIRI-195 to P.Aw.; National Institutes of Health (NIH) grants UO1HG5214, RC2HG5581 and RO1MH84698 to G.R.A

R01HG4719 and R01HG3698 to G.T.M; RC2HG5552 and U01HG6513 to G.R.A. and G.T.M.; R01HG4960 and R01HG5701 to B.L.B.; U01HG5715 to C.D.B. and A.G.C.; T32GM8283 to D.Cl.; U01HG5208 to M.J.D.; U01HG6569 to M.A.D.; R01HG2898 and R01CA166661 to S.E.D. UO1HG5209, U01HG5725 and P41HG4221 to C.Le. P01HG4120 to E.E.E.; U01HG5728 to Yu.F.; U54HG3273 and U01HG5211 to R.A.G.; 
R01HL95045 to S.B.G. U41HG4568 to S.JK. P41HG2371 to W.J.K. ES015794 Al077439, HL088133 and HL078885 to E.G.B.; RC2HL102925 to S.B.G. and D.M.A.; R01GM59290 to L.B.J and M.A.B.; U54HG3067 to E.S.L and S.B.G.; T15LM7033 to B.K.M.; T32HL94284 to J.L.R.-F.: DP2OD6514 and BAA-NIAID-DAIT-NIHAI2009061 to P.C.S.; T32GM7748 to X.S.; U54HG3079 to R.K.W.; UL1RR024131 to R.D.H.; HHSN268201100040C to the Coriell Institute for Medical Research; a Sandler Foundation award and an American Asthma Foundation award to E.G.B.; an IBM Open Collaborative Research Program award to Y.B.; an A.G. Leventis Foundation scholarship to D.K.X.; a Wolfson Royal Society Merit Award to P.Do.; a Howard Hughes Medical Institute International Fellowship award to P.H.S.; a grant from T. and V. Stanley to S.C.Y.; and a Mary Beryl Patch Turnbull Scholar Program award to K.C.B. E.H. is a faculty fellow of the Edmond J. Safra Bioinformatics program at Tel-Aviv University. E.E.E. and D.H. are investigators of the Howard Hughes Medical Institute. M.V.G. is a long-term fellow of EMBO.

Author Contributions Details of author contributions can be found in the author list.

Author Information All primary data, alignments, individual call sets, consensus call sets, integrated haplotypes with genotype likelihoods and supporting data including details of validation are available from the project website (http:// www.1000genomes.org). Variant and haplotypes for specific genomic regions and specific samples can be viewed and downloaded through the project browser (http:// browser.1000genomes.org/). Common project variants with no known medical impact have been compiled by dbSNP for filtering (http://www.ncbi.nlm.nih.gov/variation/ docs/human variation vcf/). The authors declare competing financial interests: details are available in the online version of the paper. Reprints and permissions information is available at www.nature.com/reprints. Readers are welcome to comment on the online version of the paper. Correspondence and requests for materials should be addressed to G.A.M. (mcvean@well.ox.ac.uk). This work is licensed under a Creative Commons Attribution-NonCommercial-ShareAlike 3.0 Unported licence. To view a copy of this licence, visit http://creativecommons.org/licenses/ by-nc-sa/3.0/

The 1000 Genomes Consortium (Participants are arranged by project role, then by institution alphabetically, and finally alphabetically within institutions except for Principal Investigators and Project Leaders, as indicated.)

Corresponding author Gil A. McVean ${ }^{1,2}$

Steering committee David M. Altshuler ${ }^{3,4,5}$ (Co-Chair), Richard M. Durbin ${ }^{6}$ (Co-Chair) Gonçalo R. Abecasis ${ }^{7}$, David R. Bentley ${ }^{8}$, Aravinda Chakravarti ${ }^{9}$, Andrew G. Clark ${ }^{10}$, Peter Donnelly ${ }^{1,2}$, Evan E. Eichler ${ }^{11}$, Paul Flicek ${ }^{12}$, Stacey B. Gabriel ${ }^{3}$, Richard A. Gibbs $^{13}$, Eric D. Green ${ }^{14}$, Matthew E. Hurles ${ }^{6}$. Bartha M. Knoppers ${ }^{15}$, Jan O. Korbel ${ }^{16}$ Eric S. Lander ${ }^{3}$, Charles Lee ${ }^{17}$, Hans Lehrach ${ }^{18,19}$, Elaine R. Mardis ${ }^{20}$, Gabor T. Marth $^{21}$ Gil A. McVean ${ }^{1,2}$, Deborah A. Nickerson ${ }^{22}$, Jeanette P. Schmidt ${ }^{23}$, Stephen T. Sherry ${ }^{24}$ Jun Wang ${ }^{25,26,27}$, Richard K. Wilson ${ }^{20}$

Production group: Baylor College of Medicine Richard A. Gibbs ${ }^{13}$ (Principal Investigator), Huyen Dinh ${ }^{13}$, Christie Kovar ${ }^{13}$, Sandra Lee ${ }^{13}$, Lora Lewis ${ }^{13}$, Donna Muzny ${ }^{13}$, Jeff Reid ${ }^{13}$, Min Wang ${ }^{13}$; BGI-Shenzhen Jun Wang ${ }^{25,26,27}$ (Principal Investigator), Xiaodong Fang ${ }^{25}$, Xiaosen Guo ${ }^{25}$, Min Jian ${ }^{25}$, Hui Jiang ${ }^{25}$, Xin Jin ${ }^{25}$, Guoqing $\mathrm{Li}^{25}$, Jingxiang $\mathrm{Li}^{25}$, Yingrui $\mathrm{Li}^{25}$, Zhuo $\mathrm{Li}^{25}$, Xiao $\mathrm{Liu}^{25}$, Yao Lu ${ }^{25}$, Xuedi $\mathrm{Ma}^{25}$ Zhe Su ${ }^{25}$, Shuaishuai Tai ${ }^{25}$, Meifang Tang ${ }^{25}$, Bo Wang ${ }^{25}$, Guangbiao Wang ${ }^{25}$, Honglong $W^{25}$, Renhua Wu ${ }^{25}$, Ye Yin ${ }^{25}$, Wenwei Zhang ${ }^{25}$, Jiao Zhao ${ }^{25}$, Meiru Zhao ${ }^{25}$, Xiaole Zheng ${ }^{25}$, Yan Zhou ${ }^{25}$; Broad Institute of MIT and Harvard Eric S. Lander ${ }^{3}$ (Principal Investigator), David M. Altshuler ${ }^{3,4,5}$, Stacey B. Gabrie| ${ }^{3}$ (Co-Chair), Namrata Gupta ${ }^{3}$; European Bioinformatics Institute Paul Flicek ${ }^{12}$ (Principal Investigator), Laura Clarke $^{12}$, Rasko Leinonen ${ }^{12}$, Richard E. Smith ${ }^{12}$, Xiangqun Zheng-Bradley ${ }^{12}$; Illumina David R. Bentley ${ }^{8}$ (Principal Investigator), Russell Grocock ${ }^{8}$, Sean Humphray ${ }^{8}$, Terena James $^{8}$, Zoya Kingsbury'; Max Planck Institute for Molecular Genetics Hans Lehrach ${ }^{18,19}$ (Principal Investigator), Ralf Sudbrak ${ }^{18}$ (Project Leader), Marcus W. Albrecht ${ }^{28}$, Vyacheslav S. Amstislavskiy ${ }^{18}$, Tatiana A. Borodina ${ }^{28}$, Matthias Lienhard ${ }^{18}$, Florian Mertes ${ }^{18}$, Marc Sultan ${ }^{18}$, Bernd Timmermann ${ }^{18}$, Marie-Laure Yaspo ${ }^{18}$; US National Institutes of Health Stephen T. Sherry ${ }^{24}$ (Principal Investigator); University of Oxford Gil A. McVean 1,2 (Principal Investigator); Washington University in St Louis Elaine R. Mardis ${ }^{20}$ (Co-Principal Investigator) (Co-Chair), Richard K. Wilson ${ }^{20}$ (Co-Principal Investigator), Lucinda Fulton ${ }^{20}$, Robert Fulton ${ }^{20}$, George M. Weinstock ${ }^{20}$; Wellcome Trust Sanger Institute Richard M. Durbin ${ }^{6}$ (Principal Investigator), Senduran Balasubramaniam ${ }^{6}$, John Burton ${ }^{6}$, Petr Danecek ${ }^{6}$, Thomas M. Keane ${ }^{6}$, Anja Kolb-Kokocinski $^{6}$, Shane McCarthy ${ }^{6}$, James Stalker ${ }^{6}$, Michael Quail ${ }^{6}$

Analysis group: Affymetrix Jeanette P. Schmidt ${ }^{23}$ (Principal Investigator), Christopher J. Davies ${ }^{23}$, Jeremy Gollub ${ }^{23}$, Teresa Webster ${ }^{23}$, Brant Wong ${ }^{23}$, Yiping Zhan ${ }^{23}$; Albert Einstein College of Medicine Adam Auton ${ }^{29}$ (Principal Investigator); Baylor College of Medicine Richard A. Gibbs ${ }^{13}$ (Principal Investigator), Fuli Yu ${ }^{13}$ (Project Leader), Matthew Bainbridge $e^{13}$, Danny Challis ${ }^{13}$, Uday S. Evani ${ }^{13}$, James Lu ${ }^{13}$, Donna Muzny ${ }^{13}$ Uma Nagaswamy ${ }^{13}$, Jeff Reid ${ }^{13}$, Aniko Sabo ${ }^{13}$, Yi Wang ${ }^{13}$, Jin Yu ${ }^{13}$; BGI-Shenzhen Jun Wang ${ }^{25,26,27}$ (Principal Investigator), Lachlan J. M. Coin ${ }^{25}$, Lin Fang ${ }^{25}$, Xiaosen Guo ${ }^{25}$, Xin $\mathrm{Jin}^{25}$, Guoqing $\mathrm{Li}^{25}$, Qibin $\mathrm{Li}^{25}$, Yingrui $\mathrm{Li}^{25}$, Zhenyu $\mathrm{Li}^{25}$, Haoxiang $\mathrm{Lin}^{25}$, Binghang Liu $^{25}$, Ruibang Luo ${ }^{25}$, Nan Qin ${ }^{25}$, Haojing Shao ${ }^{25}$, Bingqiang Wang ${ }^{25}$, Yinlong Xie ${ }^{25}$ Chen $\mathrm{Ye}^{25}$, Chang Yu ${ }^{25}$, Fan Zhang ${ }^{25}$, Hancheng Zheng ${ }^{25}$, Hongmei Žhu ${ }^{25}$; Boston College Gabor T. Marth ${ }^{21}$ (Principal Investigator), Erik P. Garrison ${ }^{21}$, Deniz Kural ${ }^{21}$ Wan-Ping Lee ${ }^{21}$, Wen Fung Leong ${ }^{21}$, Alistair N. Ward ${ }^{21}$, Jiantao Wu ${ }^{21}$, Mengyao
Zhang ${ }^{21}$; Brigham and Women's Hospital Charles Lee ${ }^{17}$ (Principal Investigator), Lauren Griffin ${ }^{17}$, Chih-Heng Hsieh ${ }^{17}$, Ryan E. Mills ${ }^{17,30}$, Xinghua Shi ${ }^{17}$, Marcin von Grotthuss $^{17}$, Chengsheng Zhang ${ }^{17}$; Broad Institute of MIT and Harvard Mark J. Daly ${ }^{3}$ (Principal Investigator), Mark A. DePristo ${ }^{3}$ (Project Leader), David M. Altshuler ${ }^{3,4,5}$, Eric Banks $^{3}$, Gaurav Bhatia ${ }^{3}$, Mauricio O. Carneiro ${ }^{3}$, Guillermo del Angel ${ }^{3}$, Stacey B. Gabriel ${ }^{3}$, Giulio Genovese ${ }^{3}$, Namrata Gupta ${ }^{3}$, Robert E. Handsaker ${ }^{3,5}$, Chris Hartt ${ }^{3}$, Eric S. Lander ${ }^{3}$, Steven A. McCarroll ${ }^{3}$, James C. Nemesh ${ }^{3}$, Ryan E. Poplin ${ }^{3}$, Stephen F. Schaffner ${ }^{3}$, Khalid Shakir ${ }^{3}$; Cold Spring Harbor Laboratory Seungtai C. Yoon ${ }^{31}$ (Principal Investigator), Jayon Lihm ${ }^{31}$, Vladimir Makarov ${ }^{32}$; Dankook University Hanjun $\mathrm{Jin}^{33}$ (Principal Investigator), Wook Kim ${ }^{34}$, Ki Cheol Kim ${ }^{34}$; European Molecular Biology Laboratory Jan O. Korbel ${ }^{16}$ (Principal Investigator), Tobias Rausch $^{16}$; European Bioinformatics Institute Paul Flicek ${ }^{12}$ (Principal Investigator), Kathryn Beal ${ }^{12}$, Laura Clarke ${ }^{12}$, Fiona Cunningham ${ }^{12}$, Javier Herrero ${ }^{12}$, William M. McLaren ${ }^{12}$, Graham R. S. Ritchie ${ }^{12}$, Richard E. Smith ${ }^{12}$, Xiangqun Zheng-Bradley ${ }^{12}$, Cornell University Andrew G. Clark ${ }^{10}$ (Principal Investigator), Srikanth Gottipati ${ }^{35}$, Alon Keinan $^{10}$, Juan L. Rodriguez-Flores ${ }^{10}$; Harvard University Pardis C. Sabeti ${ }^{3,36}$ (Principal Investigator), Sharon R. Grossman ${ }^{3,36}$, Shervin Tabrizi ${ }^{3,36}$, Ridhi Tariyal ${ }^{3,36}$; Human Gene Mutation Database David N. Cooper ${ }^{37}$ (Principal Investigator), Edward V' Ball $^{37}$, Peter D. Stenson ${ }^{37}$; Illumina David R. Bentley (Principal Investigator), Bret Barnes $^{38}$, Markus Bauer ${ }^{8}$, R. Keira Cheetham ${ }^{8}$, Tony Cox $^{8}$, Michael Eberle ${ }^{8}$, Sean Humphray ${ }^{8}$, Scott Kahn ${ }^{38}$, Lisa Murray ${ }^{8}$, John Peden ${ }^{8}$, Richard Shaw ${ }^{8}$; Leiden University Medical Center Kai Ye ${ }^{39}$ (Principal Investigator); Louisiana State University Mark A. Batzer ${ }^{40}$ (Principal Investigator), Miriam K. Konkel ${ }^{40}$, Jerilyn A. Walker ${ }^{40}$; Massachusetts General Hospital Daniel G. MacArthur ${ }^{41}$ (Principal Investigator), Monkol Lek ${ }^{41}$; Max Planck Institute for Molecular Genetics Ralf Sudbrak ${ }^{18}$ (Project Leader), Vyacheslav S. Amstislavskiy ${ }^{18}$, Ralf Herwig ${ }^{18}$; Pennsylvania State University Mark D. Shriver ${ }^{42}$ (Principal Investigator); Stanford University Carlos D. Bustamante ${ }^{43}$ (Principal Investigator), Jake K. Byrnes ${ }^{44}$, Francisco M. De La Vega ${ }^{10}$, Simon Gravel ${ }^{43}$ Eimear E. Kenny ${ }^{43}$, Jeffrey M. Kidd ${ }^{43}$, Phil Lacroute ${ }^{43}$, Brian K. Maples ${ }^{43}$, Andres Moreno-Estrada ${ }^{43}$, Fouad Zakharia ${ }^{43}$; Tel-Aviv University Eran Halperin ${ }^{45,46,47}$ (Principal Investigator), Yael Baran ${ }^{45}$; Translational Genomics Research Institute David W. Craig ${ }^{48}$ (Principal Investigator), Alexis Christoforides ${ }^{48}$, Nils Homer ${ }^{49}$, Tyler Izatt ${ }^{48}$, Ahmet A. Kurdoglu ${ }^{48}$, Shripad A. Sinari ${ }^{48}$, Kevin Squire ${ }^{50}$; US National Institutes of Health Stephen T. Sherry ${ }^{24}$ (Principal Investigator), Chunlin Xiao ${ }^{24}$; University of California, San Diego Jonathan Sebat ${ }^{51,52}$ (Principal Investigator), Vineet Bafna ${ }^{53}$, Kenny $\mathrm{Ye}^{54}$; University of California, San Francisco Esteban G. Burchard ${ }^{55}$ (Principal Investigator), Ryan D. Hernandez $z^{55}$ (Principal Investigator), Christopher R. Gignoux ${ }^{55}$; University of California, Santa Cruz David Haussler ${ }^{56,57}$ (Principal Investigator), Sol J. Katzman ${ }^{56}$, W. James Kent ${ }^{56}$; University of Chicago Bryan Howie ${ }^{58}$ University College London Andres Ruiz-Linares ${ }^{59}$ (Principal Investigator); University of Geneva Emmanouil T. Dermitzakis ${ }^{60,61,62}$ (Principal Investigator), Tuuli Lappalainen ${ }^{60,61,62}$; University of Maryland School of Medicine Scott E. Devine ${ }^{63}$ (Principal Investigator), Xinyue Liu ${ }^{63}$, Ankit Maroo ${ }^{63}$, Luke J. Tallon ${ }^{63}$; University of Medicine and Dentistry of New Jersey Jeffrey A. Rosenfeld ${ }^{64,65}$ (Principal Investigator), Leslie P. Michelson ${ }^{64}$; University of Michigan Gonçalo R. Abecasis ${ }^{7}$ (Principal Investigator) (Co-Chair), Hyun Min Kang (Project Leader), Paul Anderson ${ }^{7}$, Andrea Angius ${ }^{66}$, Abigail Bigham ${ }^{67}$, Tom Blackwell7 ${ }^{7}$, Fabio Busonero, $7,66,68$, Francesco Cucca ${ }^{66,68}$, Christian Fuchsberger ${ }^{7}$, Chris Jones ${ }^{69}$, Goo Jun ${ }^{7}$, Yun Li ${ }^{70}$, Robert Lyons ${ }^{71}$, Andrea Maschio $7,66,68$, Eleonora Porcu $7,66,68$, Fred Reinier ${ }^{69}$, Serena Sanna ${ }^{66}$, David Schlessinger ${ }^{72}$, Carlo Sidore $7,66,68$, Adrian Tan ${ }^{7}$, Mary Kate Trost'; University of Montréal Philip Awadalla ${ }^{73}$ (Principal Investigator), Alan Hodgkinson ${ }^{73}$; University of Oxford Gerton Lunter ${ }^{1}$ (Principal Investigator), Gil A. McVean ${ }^{1,2}$ (Principal Investigator) (Co-Chair), Jonathan L. Marchini ${ }^{1,2}$ (Principal Investigator), Simon Myers ${ }^{1,2}$ (Principal Investigator), Claire Churchhouse ${ }^{2}$, Olivier Delaneau ${ }^{2}$, Anjali Gupta-Hinch ${ }^{1}$, Zamin Iqbal ${ }^{1}$, Iain Mathieson ${ }^{1}$, Andy Rimmer ${ }^{1}$, Dionysia K. Xifara ${ }^{1,2}$; University of Puerto Rico Taras K. Oleksyk ${ }^{74}$ (Principal Investigator); University of Texas Health Sciences Center at Houston Yunxin $\mathrm{Fu}^{75}$ (Principal Investigator), Xiaoming Liü ${ }^{75}$, Momiao Xiong ${ }^{75}$; University of Utah Lynn Jorde ${ }^{76}$ (Principal Investigator), David Witherspoon ${ }^{76}$, Jinchuan Xing ${ }^{77}$; University of Washington Evan E. Eichler ${ }^{11}$ (Principal Investigator), Brian L. Browning ${ }^{78}$ (Principal Investigator), Can Alkan ${ }^{22,79}$, Iman Hajirasoulina ${ }^{80}$, Fereydoun Hormozdiari2 ${ }^{22}$, Arthur $\mathrm{Ko}^{22}$, Peter H. Sudmant ${ }^{22}$; Washington University in St Louis Elaine R. Mardis ${ }^{20}$ (Co-Principal Investigator), Ken Chen ${ }^{81}$, Asif Chinwalla ${ }^{20}$, Li Ding $^{20}$, David Dooling ${ }^{20}$, Daniel C. Koboldt ${ }^{20}$, Michael D. McLellan ${ }^{20}$, John W. Wallis ${ }^{20}$ Michael C. Wend ${ }^{20}$, Qunyuan Zhang ${ }^{20}$; Wellcome Trust Sanger Institute Richard M. Durbin $^{6}$ (Principal Investigator), Matthew E. Hurles ${ }^{6}$ (Principal Investigator), Chris Tyler-Smith $^{6}$ (Principal Investigator), Cornelis A. Albers ${ }^{82}$, Qasim Ayub ${ }^{6}$, Senduran Balasubramaniam ${ }^{6}$, Yuan Chen ${ }^{6}$. Alison J. Coffey ${ }^{6}$. Vincenza Colonna ${ }^{6,83}$, Petr Danecek $^{6}$, Ni Huang ${ }^{6}$, Luke Jostins 6 , Thomas M. Keane ${ }^{6}$, Heng Li ${ }^{3,6}$, Shane McCarthy ${ }^{6}$, Aylwyn Scally ${ }^{6}$, James Stalker ${ }^{6}, K^{2}$ Kaudia Walter ${ }^{6}$, Yali Xue ${ }^{6}$, Yujun Zhang ${ }^{6} ;$ Yale University Mark B. Gerstein ${ }^{84,85,86}$ (Principal Investigator), Alexej Abyzov ${ }^{84,86}$ Suganthi Balasubramanian ${ }^{86}$, Jieming Chen ${ }^{84}$, Declan Clarke ${ }^{87}$, Yao Fu ${ }^{84}$, Lukas Habegger ${ }^{84}$, Arif O. Harmanci ${ }^{84}$, Mike Jin ${ }^{86}$, Ekta Khurana ${ }^{86}$, Xinmeng Jasmine Mu ${ }^{84}$ Cristina Sisu ${ }^{84}$

Structural variation group: BGI-Shenzhen Yingrui $\mathrm{Li}^{25}$, Ruibang $\mathrm{LuO}^{25}$, Hongmei $\mathrm{Zhu}^{25}$; Brigham and Women's Hospital Charles Lee ${ }^{17}$ '(Principal Investigator) (Co-Chair), Lauren Griffin ${ }^{17}$, Chih-Heng Hsieh ${ }^{17}$, Ryan E. Mills ${ }^{17,30}{ }^{1}$, Xinghua Shi ${ }^{17}$ Marcin von Grotthuss ${ }^{17}$. Chengsheng Zhang ${ }^{17}$; Boston College Gabor T. Marth ${ }^{21}$ (Principal Investigator), Erik P. Garrison 21 , Deniz Kural ${ }^{21}$, Wan-Ping Lee ${ }^{21}$, Alistair N. Ward $^{21}$, Jiantao Wu ${ }^{21}$, Mengyao Zhang ${ }^{21}$; Broad Institute of MIT and Harvard Steven A. McCarroll ${ }^{3}$ (Project Leader), David M. Altshuler ${ }^{3,4,5}$, Eric Banks ${ }^{3}$, Guillermo del Angel $^{3}$, Giulio Genovese ${ }^{3}$, Robert E. Handsaker ${ }^{3,5}$. Chris Hart| $\left.\right|^{3}$, James C. Nemesh ${ }^{3}$, Khalid Shakir'; Cold Spring Harbor Laboratory Seungtai C. Yoon ${ }^{31}$ (Principal Investigator), Jayon Lihm ${ }^{31}$, Vladimir Makarov ${ }^{32}$; Cornell University Jeremiah Degenhardt ${ }^{10}$; European Bioinformatics Institute Paul Flicek ${ }^{12}$ (Principal Investigator), Laura Clarke ${ }^{12}$, Richard E. Smith ${ }^{12}$, Xiangqun Zheng-Bradley ${ }^{12}$; 
European Molecular Biology Laboratory Jan O. Korbel ${ }^{16}$ (Principal Investigator) (Co-Chair), Tobias Rausch ${ }^{16}$, Adrian M. Stütz ${ }^{16}$; Illumina David R. Bentley ${ }^{8}$ (Principal Investigator), Bret Barnes ${ }^{38}$, R. Keira Cheetham ${ }^{8}$, Michael Eberle ${ }^{8}$, Sean Humphray ${ }^{8}$ Scott Kahn ${ }^{38}$, Lisa Murray ${ }^{8}$, Richard Shaw ${ }^{8}$; Leiden University Medical Center Kai Ye ${ }^{39}$ (Principal Investigator); Louisiana State University Mark A. Batzer ${ }^{40}$ (Principal Investigator), Miriam K. Konke ${ }^{40}$, Jerilyn A. Walker ${ }^{40}$; Stanford University Phil Lacroute $^{43}$; Translational Genomics Research Institute David W. Craig ${ }^{48}$ (Principal Investigator), Nils Homer ${ }^{49}$; US National Institutes of Health Deanna Church ${ }^{24}$, Chunlin Xiao ${ }^{24}$; University of California, San Diego Jonathan Sebat ${ }^{51,52}$ (Principal Investigator), Vineet Bafna ${ }^{53}$, Jacob J. Michaelson ${ }^{88}$, Kenny Ye ${ }^{54}$; University of Maryland School of Medicine Scott E. Devine ${ }^{63}$ (Principal Investigator), Xinyue Liu ${ }^{63}$ Ankit Maro ${ }^{63}$, Luke J. Tallon ${ }^{63}$; University of Oxford Gerton Lunter ${ }^{1}$ (Principal Investigator), Gil A. McVean ${ }^{1,2}$ (Principal Investigator), Zamin Iqbal ${ }^{1}$; University of Utah David Witherspoon ${ }^{76}$, Jinchuan Xing ${ }^{77}$; University of Washington Evan E. Eichler ${ }^{11}$ (Principal Investigator) (Co-Chair), Can Alkan ${ }^{22,79}$, Iman Hajirasouliha ${ }^{80}$, Fereydoun Hormozdiari ${ }^{22}$, Arthur $\mathrm{Ko}^{22}$, Peter H. Sudmant ${ }^{22}$; Washington University in St Louis Ken Chen ${ }^{81}$, Asif Chinwalla ${ }^{20}$, Li Ding ${ }^{20}$, Michael D. McLellan ${ }^{20}$, John W. Wallis ${ }^{20}$ Wellcome Trust Sanger Institute Matthew E. Hurles ${ }^{6}$ (Principal Investigator) (Co-Chair), Ben Blackburne ${ }^{6}$, Heng Li $\mathrm{i}^{3,6}$. Sarah J. Lindsay ${ }^{6}$, Zemin Ning ${ }^{6}$, Aylwyn Scally ${ }^{6}$, Klaudia Walter ${ }^{6}$, Yujun Zhang
(Principal Investigator), Alexej Abyzov $v^{4,86}$, Jieming Chen ${ }^{84}$, Declan Clarke ${ }^{87}$, Ekta Khurana ${ }^{86}$, Xinmeng Jasmine $\mathrm{Mu}^{84}$, Cristina Sisu ${ }^{84}$

Exome group: Baylor College of Medicine Richard A. Gibbs ${ }^{13}$ (Principal Investigator) (Co-Chair), Fuli Yu ${ }^{13}$ (Project Leader). Matthew Bainbridge ${ }^{13}$, Danny Challis ${ }^{13}$, Uday S. Evani $^{13}{ }^{13}$, Christie Kovar ${ }^{13}$, Lora Lewis ${ }^{13}$, James Lu ${ }^{13}$, Donna Muzny ${ }^{13}$, Uma Nagaswamy ${ }^{13}$, Jeff Reid ${ }^{13}$, Aniko Sabo ${ }^{13}$, Jin Yu ${ }^{13}$; BGI-Shenzhen Xiaosen Guo ${ }^{25}$, Yingrui $\mathrm{Li}^{25}$, Renhua $\mathrm{Wu}^{25}$; Boston College Gabor T. Marth ${ }^{21}$ (Principal Investigator) (Co-Chair), Erik P. Garrison ${ }^{21}$, Wen Fung Leong ${ }^{21}$, Alistair N. Ward ${ }^{21}$; Broad Institute of MIT and Harvard Guillermo del Angel ${ }^{3}$, Mark A. DePristo ${ }^{3}$, Stacey B. Gabriel ${ }^{3}$, Namrata Gupta $^{3}$, Chris Hartl ${ }^{3}$, Ryan E. Poplin ${ }^{3}$; Cornell University Andrew G. Clark ${ }^{10}$ (Principal Investigator), Juan L. Rodriguez-Flores ${ }^{10}$; European Bioinformatics Institute Paul Flicek $^{12}$ (Principal Investigator), Laura Clarke ${ }^{12}$, Richard E. Smith ${ }^{12}$, Xiangqun Zheng-Bradley 12; Massachusetts General Hospital Daniel G. MacArthur ${ }^{41}$ (Principal Investigator); Stanford University Carlos D. Bustamante ${ }^{43}$ (Principal Investigator), Simon Gravel $^{43}$; Translational Genomics Research Institute David W. Craig ${ }^{48}$ (Principal Investigator), Alexis Christoforides ${ }^{48}$, Nils Homer ${ }^{49}$, Tyler Izatt ${ }^{48}$; US National Institutes of Health Stephen T. Sherry ${ }^{24}$ (Principal Investigator), Chunlin $X_{i a 0^{24}} ;$ University of Geneva Emmanouil T. Dermitzakis ${ }^{60,61,62}$ (Principal Investigator); University of Michigan Gonçalo R. Abecasis ${ }^{7}$ (Principal Investigator), Hyun Min Kang University of Oxford Gil A. McVean ${ }^{1,2}$ (Principal Investigator); Washington University in St Louis Elaine R. Mardis ${ }^{20}$ (Principal Investigator), David Dooling ${ }^{20}$, Lucinda Fulton ${ }^{20}$ Robert Fulton ${ }^{20}$, Daniel C. Koboldt ${ }^{20}$; Wellcome Trust Sanger Institute Richard M. Durbin $^{6}$ (Principal Investigator), Senduran Balasubramaniam ${ }^{6}$. Thomas M. Keane ${ }^{6}$ Shane McCarthy ${ }^{6}$, James Stalker ${ }^{6}$; Yale University Mark B. Gerstein ${ }^{84,85,86}$ (Principal Investigator), Suganthi Balasubramanian ${ }^{86}$, Lukas Habegger ${ }^{84}$

Functional interpretation group: Boston College Erik P. Garrison ${ }^{21}$; Baylor College of Medicine Richard A. Gibbs $s^{13}$ (Principal Investigator), Matthew Bainbridge ${ }^{13}$, Donna Muzny ${ }^{13}$, Fuli Yu ${ }^{13}$, Jin Yu${ }^{13}$; Broad Institute of MIT and Harvard Guillermo del Angel ${ }^{3}$, Robert E. Handsaker ${ }^{3,5}$; Cold Spring Harbor Laboratory Vladimir Makarov ${ }^{32}$; Cornell University Juan L. Rodriguez-Flores ${ }^{10}$; Dankook University Hanjun Jin ${ }^{33}$ (Principal Investigator), Wook Kim ${ }^{34}$ Ki Cheol Kim³. European Bioinformatics Institute Paul Flicek $^{12}$ (Principal Investigator), Kathryn Beal ${ }^{12}$, Laura Clarke ${ }^{12}$, Fiona Cunningham ${ }^{12}$ Javier Herrero ${ }^{12}$, William M. McLaren ${ }^{12}$, Graham R. S. Ritchie ${ }^{12}$, Xiangqun Zheng-Bradley ${ }^{12}$; Harvard University Shervin Tabrizi ${ }^{3,36}$, Massachusetts General Hospital Daniel G. MacArthur ${ }^{41}$ (Principal Investigator), Monkol Lek ${ }^{41}$; Stanford University Carlos D. Bustamante ${ }^{43}$ (Principal Investigator), Francisco M. De La Vega ${ }^{10}$ Translational Genomics Research Institute David W. Craig ${ }^{48}$ (Principal Investigator), Ahmet A. Kurdoglu ${ }^{48}$; University of Geneva Tuuli Lappalainen ${ }^{60,61,62}$; University of Medicine and Dentistry of New Jersey Jeffrey A. Rosenfeld ${ }^{64,65}$ (Principal Investigator), Leslie P. Michelson ${ }^{64,65}$; University of Montréal Philip Awadalla ${ }^{73}$ (Principal Investigator), Alan Hodgkinson ${ }^{73}$; University of Oxford Gil A. McVean ${ }^{1,2}$ (Principal Investigator); Washington University in St Louis Ken Chen ${ }^{81}$; Wellcome Trust Sanger Institute Chris Tyler-Smith ${ }^{6}$ (Principal Investigator) (Co-Chair), Yuan Chen $^{6}$, Vincenza Colonna ${ }^{6,83}$, Adam Frankish ${ }^{6}$, Jennifer Harrow ${ }^{6}$, Yali Xue ${ }^{6}$; Yale University Mark B. Gerstein ${ }^{84,85,86}$ (Principal Investigator) (Co-Chair), Alexej Abyzov $^{84,86}$, Suganthi Balasubramanian ${ }^{86}$, Jieming Chen ${ }^{84}$, Declan Clarke ${ }^{87}$, Yao Fu ${ }^{84}$ Arif O. Harmanci ${ }^{84}$, Mike Jin ${ }^{86}$, Ekta Khurana ${ }^{86}$, Xinmeng Jasmine Mu ${ }^{84}$, Cristina Sisu ${ }^{84}$

Data coordination centre group: Baylor College of Medicine Richard A. Gibbs ${ }^{13}$ (Principal Investigator), Gerald Fowler ${ }^{13}$, Walker Hale ${ }^{13}$, Divya Kalra ${ }^{13}$, Christie Kovar $^{13}$, Donna Muzny ${ }^{13}$, Jeff Reid ${ }^{13}$; BGI-Shenzhen Jun Wang ${ }^{25,26,27}$ (Principal Investigator), Xiaosen Guo ${ }^{25}$, Guoqing $\mathrm{Li}^{25}$, Yingrui $\mathrm{Li}^{25}$, Xiaole Zheng ${ }^{25}$; Broad Institute of MIT and Harvard David M. Altshuler ${ }^{3,4,5}$; European Bioinformatics Institute Paul Flicek ${ }^{12}$ (Principal Investigator) (Co-Chair), Laura Clarke ${ }^{12}$ (Project Leader), Jonathan Barker ${ }^{12}$ Gavin Kelman ${ }^{12}$, Eugene Kulesha ${ }^{12}$, Rasko Leinonen ${ }^{12}$, William M. McLaren ${ }^{12}$, Rajesh Radhakrishnan ${ }^{12}$, Asier Roa ${ }^{12}$, Dmitriy Smirnov ${ }^{12}$, Richard E. Smith ${ }^{12}$, lan Streeter ${ }^{12}$ Iliana Toneva ${ }^{12}$, Brendan Vaughan ${ }^{12}$, Xiangqun Zheng-Bradley ${ }^{12}$; Illumina David R. Bentley ${ }^{8}$ (Principal Investigator), Tony Cox ${ }^{8}$, Sean Humphray ${ }^{8}$, Scott Kahn ${ }^{38}$; Max Planck Institute for Molecular Genetics Ralf Sudbrak ${ }^{18}$ (Project Leader), Marcus W. Albrecht ${ }^{28}$, Matthias Lienhard ${ }^{18}$; Translational Genomics Research Institute David W. $\mathrm{Craig}^{48}$ (Principal Investigator), Tyler Izatt ${ }^{48}$, Ahmet A. Kurdoglu ${ }^{48}$; US National Institutes of Health Stephen T. Sherry ${ }^{24}$ (Principal Investigator) (Co-Chair), Victor Ananiev $^{24}$, Zinaida Belaia ${ }^{24}$, Dimitriy Beloslyudtsev ${ }^{24}$, Nathan Bouk ${ }^{24}$. Chao Chen ${ }^{24}$ Deanna Church ${ }^{24}$, Robert Cohen ${ }^{24}$, Charles Cook ${ }^{24}$, John Garner ${ }^{24}$, Timothy
Hefferon ${ }^{24}$ Mikhail Kimelman ${ }^{24}$ Chunlei Liu ${ }^{24}$ John Lopez ${ }^{24}$ Peter Meric ${ }^{24}$ Chris O'Sullivan $^{89}$, Yuri Ostapchuk ${ }^{24}$, Lon Phan ${ }^{24}$, Sergiy Ponomarov ${ }^{24}$, Valerie Schneider ${ }^{24}$ Eugene Shekhtman ${ }^{24}$, Karl Sirotkin ${ }^{24}$, Douglas Slotta ${ }^{24}$, Chunlin Xiao ${ }^{24}$, Hua Zhang ${ }^{24}$ University of California, Santa Cruz David Haussler ${ }^{56,57}$ (Principal Investigator); University of Michigan Gonçalo R. Abecasis ${ }^{7}$ (Principal Investigator); University of Oxford Gil A. McVean 1,2 (Principal Investigator); University of Washington Can Alkan $^{22,79}$, Arthur Ko ${ }^{22}$; Washington University in St Louis David Dooling ${ }^{20}$; Wellcome Trust Sanger Institute Richard M. Durbin ${ }^{6}$ (Principal Investigator), Senduran Balasubramaniam ${ }^{6}$, Thomas M. Keane ${ }^{6}$, Shane McCarthy ${ }^{6}$, James Stalker ${ }^{6}$

Samples and ELSI group Aravinda Chakravarti ${ }^{9}$ (Co-Chair), Bartha M. Knoppers ${ }^{15}$ (Co-Chair), Gonçalo R. Abecasis ${ }^{7}$, Kathleen C. Barnes ${ }^{9}$, Christine Beiswanger ${ }^{91}$ Esteban G. Burchard ${ }^{55}$, Carlos D. Bustamante ${ }^{43}$, Hongyu Cai ${ }^{25}$, Hongzhi Cao $^{25}$, Richard M. Durbin ${ }^{6}$, Neda Gharani $i^{91}$, Richard A. Gibbs ${ }^{13}$, Christopher R. Gignoux ${ }^{55}$, Simon Grave $^{43}$, Brenna Henn ${ }^{43}$, Danielle Jones ${ }^{35}$, Lynn Jorde ${ }^{76}$, Jane S. Kaye ${ }^{92}$, Alon Keinan $^{10}$, Alastair Kent ${ }^{93}$, Angeliki Kerasidou ${ }^{1}$, Yingrui Li ${ }^{25}$, Rasika Mathias ${ }^{94}$, Gil A. McVean $^{1,2}$, , Andres Moreno-Estrada ${ }^{43}$, Pilar N. Ossorio ${ }^{55,96}$, Michael Parker ${ }^{97}$, David Reich $^{5}$, Charles N. Rotimi ${ }^{98}$, Charmaine D. Royal ${ }^{99}$, Karla Sandoval ${ }^{43}$, Yeyang Su ${ }^{25}$, Ralf Sudbrak ${ }^{18}$, Zhongming Tian ${ }^{25}$, Bernd Timmermann ${ }^{18}$, Sarah Tishkoff ${ }^{100}$, Lorraine H. Toji $^{11}{ }^{2}$ Chris Tyler-Smith ${ }^{6}$, Marc Via ${ }^{101}$, Yuhong Wang ${ }^{25}$, Huanming Yang ${ }^{25}$, Ling Yang ${ }^{25}$, Jiayong Zhu ${ }^{25}$

Sample collection: British from England and Scotland (GBR) Walter Bodmer ${ }^{102 .}$ Colombians in Medellín, Colombia (CLM) Gabriel Bedoya ${ }^{103}$, Andres Ruiz-Linares ${ }^{59}$ Han Chinese South (CHS) Cai Zhi Ming ${ }^{25}$, Gao Yang ${ }^{104}$, Chu Jia You ${ }^{105}$; Finnish in Finland (FIN) Leena Peltonent; Iberian populations in Spain (IBS) Andres Garcia-Montero ${ }^{106}$, Alberto Orfao ${ }^{107}$; Puerto Ricans in Puerto Rico (PUR) Julie Dutil $^{108}$, Juan C. Martinez-Cruzado ${ }^{74}$, Taras K. Oleksyk ${ }^{74}$

Scientific management Lisa D. Brooks ${ }^{109}$, Adam L. Felsenfeld ${ }^{109}$, Jean E. McEwen ${ }^{109}$ Nicholas C. Clemm ${ }^{109}$, Audrey Duncanson ${ }^{110}$, Michael Dunn ${ }^{110}$, Eric D. Green ${ }^{14}$, Mark S. Guyer ${ }^{109}$, Jane L. Peterson ${ }^{109}$

Writing group Goncalo R. Abecasis ${ }^{7}$, Adam Auton ${ }^{29}$, Lisa D. Brooks ${ }^{109}$, Mark A. DePristo ${ }^{3}$, Richard M. Durbin ${ }^{6}$, Robert E. Handsaker ${ }^{3,5}$, Hyun Min Kang ${ }^{7}$, Gabor T. Marth $^{21}$, Gil A. McVean ${ }^{1,2}$

${ }^{1}$ Wellcome Trust Centre for Human Genetics, Oxford University, Oxford OX3 7BN, UK ${ }^{2}$ Department of Statistics, Oxford University, Oxford OX1 3TG, UK. ${ }^{3}$ The Broad Institute of MIT and Harvard, 7 Cambridge Center, Cambridge, Massachusetts 02142, USA. ${ }^{4}$ Center for Human Genetic Research, Massachusetts General Hospital, Boston, Massachusetts 02114, USA. ${ }^{5}$ Department of Genetics, Harvard Medical School, Cambridge, Massachusetts 02142, USA. ${ }^{6}$ Wellcome Trust Sanger Institute, Wellcome Trust Genome Campus, Cambridge CB10 1SA, UK. ${ }^{7}$ Center for Statistical Genetics, Biostatistics, University of Michigan, Ann Arbor, Michigan 48109, USA. ${ }^{8}$ Illumina United Kingdom, Chesterford Research Park, Little Chesterford, Near Saffron Walden, Essex CB10 1XL, UK 9McKusick-Nathans Institute of Genetic Medicine, Johns Hopkins University School of Medicine, Baltimore, Maryland 21205, USA. ${ }^{10} \mathrm{Center}$ for Comparative and Population Genomics, Cornell University, Ithaca, New York 14850, USA. ${ }^{11}$ Department of Genome Sciences, University of Washington School of Medicine and Howard Hughes Medical Institute, Seattle, Washington 98195, USA. ${ }^{12}$ European Bioinformatics Institute, Wellcome Trust Genome Campus, Cambridge CB10 1SD, UK. ${ }^{13}$ Baylor College of Medicine, Human Genome Sequencing Center, Houston, Texas 77030, USA. ${ }^{14}$ US National Institutes of Health, National Human Genome Research Institute, 31 Center Drive, Bethesda, Maryland 20892, USA. ${ }^{15}$ Centre of Genomics and Policy, McGill University, Montréal, Québec H3A 1A4, Canada. ${ }^{16}$ European Molecular Biology Laboratory, Genome Biology Research Unit, Meyerhofstraße 1, 69117 Heidelberg. Germany. ${ }^{17}$ Department of Pathology, Brigham and Women's Hospital and Harvard Medical School, Boston, Massachusetts 02115, USA. ${ }^{18}$ Max Planck Institute for Molecular Genetics, Ihnestraße 63-73, 14195 Berlin, Germany. ${ }^{19}$ Dahlem Centre for Genome Research and Medical Systems Biology, D-14195 Berlin-Dahlem, Germany. ${ }^{20}$ The Genome Center, Washington University School of Medicine, St Louis, Missouri 63108, USA. ${ }^{21}$ Department of Biology, Boston College, Chestnut Hill, Massachusetts 02467, USA. ${ }^{22}$ Department of Genome Sciences, University of Washington School of Medicine, Seattle, Washington 98195, USA. ${ }^{23}$ Affymetrix, Inc., Santa Clara, California 95051, USA. ${ }^{24}$ US National Institutes of Health, National Center for Biotechnology Information, 45 Center Drive, Bethesda, Maryland 20892, USA. ${ }^{25}$ BGI-Shenzhen, Shenzhen 518083, China. ${ }^{26}$ The Novo Nordisk Foundation Center for Basic Metabolic Research, University of Copenhagen, DK-2200 Copenhagen, Denmark. ${ }^{27}$ Department of Biology, University of Copenhagen, DK-2100 Copenhagen, Denmark. ${ }^{28}$ Alacris Theranostics GmbH, D-14195 Berlin-Dahlem, Germany. ${ }^{29}$ Department of Genetics, Albert Einstein College of Medicine Bronx, New York 10461, USA. ${ }^{30}$ Department of Computational Medicine and Bioinfomatics, University of Michigan, Ann Arbor, Michigan 48109, USA. ${ }^{31}$ Cold Spring Harbor Laboratory, Cold Spring Harbor, New York 11724, USA. ${ }^{32}$ Seaver Autism Center and Department of Psychiatry, Mount Sinai School of Medicine, New York, New York 10029 , USA. ${ }^{33}$ Department of Nanobiomedical Science, Dankook University, Cheonan 330-714, South Korea. ${ }^{34}$ Department of Biological Sciences, Dankook University, Cheonan 330-714, South Korea. ${ }^{35}$ Department of Biological Statistics and Computational Biology, Cornell University, Ithaca, New York 14853, USA. ${ }^{36}$ Center for Systems Biology and Department Organismic and Evolutionary Biology, Harvard University, Cambridge, Massachusetts 02138, USA. ${ }^{37}$ Institute of Medical Genetics, School of Medicine, Cardiff University, Heath Park, Cardiff CF14 4XN, UK ${ }^{38}$ Illumina, Inc. San Diego, California 92122, USA. ${ }^{39}$ Molecular Epidemiology Section, Department of Medical Statistics and Bioinformatics, Leiden University Medical Center 2333 ZA, The Netherlands. ${ }^{40}$ Department of Biological Sciences, Louisiana State University, Baton 
Rouge, Louisiana 70803, USA. ${ }^{41}$ Analytic and Translational Genetics Unit, Massachusetts General Hospital, Boston, Massachusetts 02114, USA. ${ }^{42}$ Department of Anthropology, Penn State University, University Park, Pennsylvania 16802, USA. ${ }^{43}$ Department of Genetics, Stanford University, Stanford, California 94305, USA. ${ }^{44}$ Ancestry.com, San Francisco, California 94107, USA. ${ }^{45}$ Blavatnik School of Computer Science, Tel-Aviv University, 69978 Tel Aviv, Israel. ${ }^{46}$ Department of Microbiology, Tel-Aviv University, 69978 Tel Aviv, Israel. ${ }^{47}$ International Computer Science Institute, Berkeley, California 94704, USA. ${ }^{48}$ The Translational Genomics Research Institute, Phoenix, Arizona 85004 , USA. ${ }^{4}$ Life Technologies, Beverly, Massachusetts 01915, USA. ${ }^{50}$ Department of Human Genetics, David Geffen School of Medicine, University of California, Los Angeles, California 90024, USA. ${ }^{51}$ Department of Psychiatry, University of California, San Diego, La Jolla, California 92093, USA. ${ }^{52}$ Department of Cellular and Molecular Medicine, University of California, San Diego, La Jolla, California 92093, USA. ${ }^{53}$ Department of Computer Science, University of California, San Diego, La Jolla, California 92093, USA. ${ }^{54}$ Department of Epidemiology and Population Health, Albert Einstein College of Medicine, Bronx, New York 10461, USA. ${ }^{55}$ Department of Bioengineering and Therapeutic Sciences and Medicine, University of California, San Francisco, California 94158, USA. ${ }^{56}$ Center for Biomolecular Science and Engineering, University of California, Santa Cruz, California 95064, USA. ${ }^{57}$ Howard Hughes Medical Institute, Santa Cruz, California 95064, USA. ${ }^{58}$ Department of Human Genetics, University of Chicago, Chicago, Illinois 60637, USA. ${ }^{59}$ Department of Genetics, Evolution and Environment, University College London, London WC1E 6BT, UK. ${ }^{60}$ Department of Genetic Medicine and Development, University of Geneva Medical School, 1211 Geneva, Switzerland. ${ }^{61}$ Institute for Genetics and Genomics in Geneva (iGE3), University of Geneva, 1211 Geneva, Switzerland. ${ }^{62}$ Swiss Institute of Bioinformatics, 1211 Geneva, Switzerland. ${ }^{63}$ Institute for Genome Sciences, University of Maryland School of Medicine, Baltimore, Maryland 21201, USA. ${ }^{64}$ IST/High Performance and Research Computing, University of Medicine and Dentistry of New Jersey, Newark, New Jersey 07107,USA. ${ }^{65}$ Department of Invertebrate Zoology, American Museum of Natural History, New York, New York 10024, USA. ${ }^{66}$ Istituto di Ricerca Genetica e Biomedica, CNR, Monserrato, 09042 Cagliari, Italy. ${ }^{67}$ Department of Anthropology, University of Michigan, Ann Arbor, Michigan 48109, USA. ${ }^{68}$ Dipartimento di Scienze Biomediche, Università delgi Studi di Sassari, 07100 Sassari, Italy. ${ }^{69} \mathrm{Center}$ for Advanced Studies, Research, and Development in Sardinia (CRS4), AGCT Program, Parco Scientifico e tecnologico della Sardegna, 09010 Pula, Italy. ${ }^{70}$ Department of Genetics, University of North Carolina at Chapel Hill, Chapel Hill, North Carolina 27599, USA. ${ }^{71}$ University of Michigan Sequencing Core, University of Michigan, Ann Arbor, Michigan 48109, USA. ${ }^{72}$ National Institute on Aging, Laboratory of Genetics, Baltimore, Maryland 21224, USA. ${ }^{73}$ Department of Pediatrics, University of Montréal, Ste. Justine Hospital Research Centre, Montréal, Québec H3T 1C5, Canada. ${ }^{74}$ Department of Biology, University of Puerto Rico, Mayagüez, Puerto Rico 00680, USA. ${ }^{75}$ The University of Texas Health Science Center at Houston, Houston, Texas 77030, USA. ${ }^{76}$ Eccles Institute of Human Genetics, University of Utah School of Medicine, Salt Lake City, Utah 84112, USA. ${ }^{77}$ Department of Genetics, Rutgers University, The State University of New Jersey, Piscataway, New Jersey 08854, USA. ${ }^{78}$ Department of Medicine, Division of Medica Genetics, University of Washington, Seattle, Washington 98195, USA. ${ }^{79}$ Department of
Computer Engineering, Bilkent University, TR-06800 Bilkent, Ankara, Turkey. ${ }^{80}$ Department of Computer Science, Simon Fraser University, Burnaby, British Columbia V5A 1S6, Canada. ${ }^{81}$ Department of Bioinformatics and Computational Biology, The University of Texas MD Anderson Cancer Center, Houston, Texas 77230, USA.

${ }^{82}$ Department of Haematology, University of Cambridge and National Health Service Blood and Transplant, Cambridge CB2 1TN, UK. ${ }^{83}$ Institute of Genetics and Biophysics, National Research Council (CNR), 80125 Naples, Italy. ${ }^{84}$ Program in Computational Biology and Bioinformatics, Yale University, New Haven, Connecticut 06520, USA. ${ }^{85}$ Department of Computer Science, Yale University, New Haven, Connecticut 06520, USA. ${ }^{86}$ Department of Molecular Biophysics and Biochemistry, Yale University, New Haven, Connecticut 06520, USA. ${ }^{87}$ Department of Chemistry, Yale University, New Haven, Connecticut 06520, USA. ${ }^{8} 8$ Beyster Center for Genomics of Psychiatric Diseases, University of California, San Diego, La Jolla, California 92093, USA. ${ }^{89}$ US National Institutes of Health, National Human Genome Research Institute, 50 South Drive, Bethesda, Maryland 20892, USA. ${ }^{90}$ Division of Allergy and Clinical Immunology, School of Medicine, Johns Hopkins University, Baltimore, Maryland 21205, USA. ${ }^{91}$ Coriell Institute for Medical Research, Camden, New Jersey 08103, USA. ${ }^{92}$ Centre for Health, Law and Emerging Technologies, University of Oxford, Oxford OX3 7LF, UK. ${ }^{93}$ Genetic Alliance, London N1 3QP, UK. ${ }^{94}$ Johns Hopkins University School of Medicine, Baltimore, Maryland 21205, USA. ${ }^{95}$ Department of Medical History and Bioethics, Morgridge Institute for Research, University of Wisconsin-Madison, Madison, Wisconsin 53706, USA.

${ }^{96}$ University of Wisconsin Law School, Madison, Wisconsin 53706, USA. ${ }^{97}$ The Ethox Centre, Department of Public Health, University of Oxford, Old Road Campus, Oxford OX3 7LF, UK. ${ }^{98}$ US National Institutes of Health, Center for Research on Genomics and Global Health, National Human Genome Research Institute, 12 South Drive, Bethesda, Maryland 20892, USA. ${ }^{99}$ Institute for Genome Sciences and Policy, Duke University, Durham, North Carolina 27708, USA. ${ }^{100}$ Department of Genetics, University of Pennsylvania School of Medicine, Philadel phia, Pennsylvania 19104, USA. ${ }^{101}$ Department of Animal Biology, Unit of Anthropology, University of Barcelona, 08028 Barcelona, Spain. ${ }^{1{ }^{2}}$ Cancer and Immunogenetics Laboratory, University of Oxford, John Radcliffe Hospital, Oxford OX3 9DS, UK. ${ }^{103}$ Laboratory of Molecular Genetics, Institute of Biology, University of Antioquia, Medellín, Colombia. ${ }^{104}$ Peking University Shenzhen Hospital, Shenzhen 518036, China. ${ }^{105}$ Institute of Medical Biology, Chinese Academy of Medical Sciences and Peking Union Medical College, Kunming 650118, China. ${ }^{106}$ Instituto de Biologia Moleculary Celular del Cancer, Centro de Investigacion del Cancer/IBMCC (CSIC-USAL), Institute of Biomedical Research of Salamanca (IBSAL) \& Banco Nacional de ADN Carlos III, University of Salamanca, 37007 Salamanca, Spain. ${ }^{107}$ Instituto de Biologia Molecular y Celular del Cancer, Centro de Investigacion del Cancer/IBMCC (CSIC-USAL), Institute of Biomedical Research of Salamanca (IBSAL) \& Cytometry Service and Department of Medicine, 37007 University of Salamanca, Salamanca, Spain. ${ }^{108}$ Ponce School of Medicine and Health Sciences, Ponce, Puerto Rico 00716, USA. ${ }^{109}$ US National Institutes of Health, National Human Genome Research Institute, 5635 Fishers Lane, Bethesda, Maryland 20892, USA. ${ }^{110}$ Wellcome Trust, Gibbs Building, 215 Euston Road, London NW1 2BE, UK.

\$Deceased. 